\title{
Total Synthesis of Basiliskamides A and B
}

\author{
Darren J. Lipomi, Neil F. Langille, and James S. Panek* \\ Department of Chemistry and Center for Chemical Methodology and Library \\ Development, Boston University, \\ 590 Commonwealth Avenue, Boston, Massachusetts 02215
}

General Information. ${ }^{1} \mathrm{H}$ NMR spectra were taken in $\mathrm{CDCl}_{3}$ at 270,300 , or $400 \mathrm{MHz}$; ${ }^{13} \mathrm{C}$ NMR spectra were taken at 67.9 or $75 \mathrm{MHz}$ as indicated. Chemical shifts are reported in parts per million using the solvent resonance internal standard $\left(\mathrm{CDCl}_{3}, 7.24\right.$ and $77.0 \mathrm{ppm}$; DMSO- $d^{6}, 2.49$ and $\left.40.0 \mathrm{ppm}\right)$. Data are reported as follows: chemical shift, multiplicity $(\mathrm{s}=$ singlet, $\mathrm{d}=$ doublet, $\mathrm{t}=$ triplet, $\mathrm{ABq}=\mathrm{AB}$ quartet, $\mathrm{m}=$ multiplet, $\mathrm{br}=$ broad par obsc $=$ partially obscured, ovlp $=$ overlapping ) and coupling constant. Infrared resonance spectra were recorded on a Nicolet Nexus ${ }^{\mathrm{TM}} 670$ FTIR spectrophotometer. High resolution mass spectra were obtained on a Finigan ${ }^{\mathrm{TM}}$ MAT-90 spectrometer. Optical rotations were recorded on an Autopol III digital polarimeter at $589 \mathrm{~nm}$ and reported as follows: $[\alpha]^{23}$, concentration $(c$ in $\mathrm{g} / 100 \mathrm{~mL})$ and solvent. All reactions were carried out in oven-dried glassware under an argon atmosphere unless stated otherwise. THF was distilled from sodium and benzophenone ketal. $\mathrm{CH}_{2} \mathrm{Cl}_{2}$ and DMSO were distilled from calcium hydride. $\mathrm{MeOH}$ and $\mathrm{PhMe}$ were obtained from a dry solvent system (alumina) and used without further drying. $\mathrm{TiCl}_{4}$ was distilled from copper powder. $\mathrm{Et}_{3} \mathrm{~N}$, DIPEA, and 2,6-lutidine were distilled from and stored over $\mathrm{KOH} .(\mathrm{COCl})_{2}$ was distilled immediately prior to use. $\mathrm{CrCl}_{2}$ was dried by brief exposure to flame under reduced pressure. All other solvents and reagents were used as supplied. Analytical thin layer chromatography was performed on Sorbent Technologies $0.20 \mathrm{~mm}$ silica gel $60 \AA$ plates. Flash chromatography ${ }^{1}$ was performed on Sorbent Technologies 32-63 $\mu \mathrm{m} 60$ Å silica gel.

${ }^{1}$ Still, W. C.; Kahn, M.; Mitra, A. J. Org. Chem. 1978, 43, 2923-2925. 
<smiles>CC(=O)CC=CC(C)[C@@H](O)CCOc1ccccc1</smiles>
(3E,5R,6S)-8-(Benzyloxy)-6-hydroxy-5-methyloct-3enoic acid methyl ester (9): A solution of 3benzyloxypropanal $^{2}(1.01 \mathrm{~g}, 6.15 \mathrm{mmol})$ and $(R, E)$ -

crotylsilane $7^{3}$ (2.08 g, 7.92 mmol, 1.3 equiv.) was prepared in $\mathrm{CH}_{2} \mathrm{Cl}_{2}$ (30.5 mL, 0.2 M) and cooled to $-78{ }^{\circ} \mathrm{C}$. The solution was treated with $\mathrm{TiCl}_{4}(0.87 \mathrm{~mL}, 7.92 \mathrm{mmol}$, 1.3 equiv.) and stirred at $-50{ }^{\circ} \mathrm{C}$ for $12 \mathrm{~h}$. The reaction was diluted with EtOAc $(5 \mathrm{~mL})$ and quenched with $\mathrm{H}_{2} \mathrm{O}(5 \mathrm{~mL})$. The cold bath was removed and the solution was poured into a seperatory funnel. The aqueous layer was extracted into EtOAc $(3 \times)$. The combined organic extracts were washed with brine, dried over $\mathrm{MgSO}_{4}$, filtered, and concentrated in vacuo. The crude material was purified by flash chromatography (silica, 20\% EtOAc/hexanes), which yielded $9(1.25 \mathrm{~g}, 74 \%)$ as a colorless oil. ${ }^{1} \mathrm{H}$ NMR (270 MHz, $\left.\mathrm{CDCl}_{3}\right) \delta$ 7.25-7.36 (m, 5H), 5.53-5.58 (m, 2H), $4.50(\mathrm{~s}, 2 \mathrm{H}), 3.59-$ 3.72 (obsc m, 3H), 3.66 (s, 3H) 3.05 (d, J = 5.4 Hz, 2H), 2.47 (br s, 1H), 2.21 (m, 1H), $1.72(\mathrm{dt}, J=5.5,6.0 \mathrm{~Hz}, 2 \mathrm{H}), 1.02(\mathrm{~d}, J=6.8 \mathrm{~Hz}, 3 \mathrm{H}) ;{ }^{13} \mathrm{C} \mathrm{NMR}\left(67.9 \mathrm{MHz}, \mathrm{CDCl}_{3}\right) \delta$ 172.4, 137.9, 128.4, 127.6, 122.7, 122.2, 74.2, 73.2, 69.1, 51.7, 42.9, 37.8, 33.7, 16.1; IR (neat) $v_{\max } 3464,3030,2954,2927,2870,1739,1455,1436,1167,1097,974 \mathrm{~cm}^{-1}$; HRMS (CI, $\mathrm{NH}_{3}$ ), $m / z$ calculated for $\mathrm{C}_{17} \mathrm{H}_{25} \mathrm{O}_{4}[\mathrm{M}+\mathrm{H}]^{+}$293.1753, found: 293.1737; $[\alpha]_{\mathrm{D}}^{23}=+7^{\circ}\left(c 2.0, \mathrm{CHCl}_{3}\right)$.

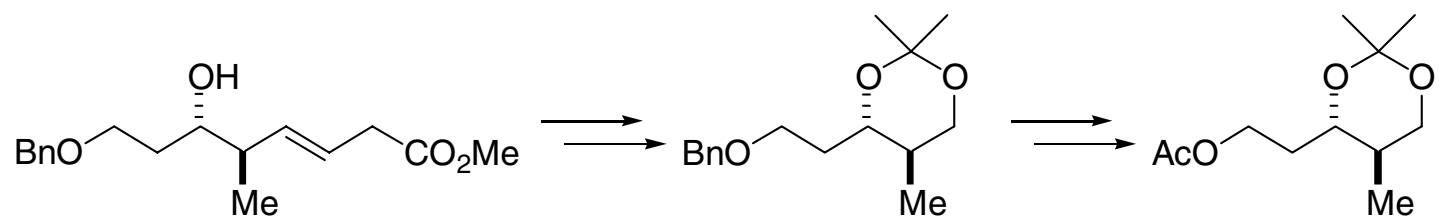

Confirmation of anti addition, acetonide (11): A solution of homoallylic alcohol (9) (326 mg, $1.19 \mathrm{mmol})$ in $\mathrm{MeOH}(27.5 \mathrm{~mL}, 0.04 \mathrm{M})$ was treated with pyridine $(0.18 \mathrm{~mL}$, $1.19 \mathrm{mmol}, 1.0$ equiv.). The solution was cooled to $-78{ }^{\circ} \mathrm{C}$ and ozone was bubbled through until the starting material was consumed as determined by TLC $(20 \%$ EtOAc/hexanes). The reaction was quenched with $\mathrm{Me}_{2} \mathrm{~S}(0.65 \mathrm{~mL}, 23.8 \mathrm{mmol}, 20.0$

\footnotetext{
${ }^{2}$ Seo, M. H.; Lee, Y. Y.; Goo, Y. M. Bull. Korean Chem. Soc. 1996, 17, 314-321.

${ }^{3}$ For preparation of chiral $(E)$-crotylsilanes, see: Beresis, R. T.; Solomon, J. S.; Yang, M. G.; Jain, N. F.; Panek, J. S.; Org. Synth. 1998, 75, 78-88.
} 
equiv.) and allowed to warm to ambient temperature over $12 \mathrm{~h}$. Volatile material was then removed under reduced pressure, and the crude residue was diluted with EtOAc. The material was washed with $\mathrm{H}_{2} \mathrm{O}(3 \times)$ and then with brine $(1 \times)$. The organic phase was dried with $\mathrm{MgSO}_{4}$, filtered, and concentrated under reduced pressure, yielding a crude aldehyde that was used without further purification in the following step. The crude aldehyde $(264 \mathrm{mg})$ was dissolved in $\mathrm{MeOH}(9.3 \mathrm{~mL}, 0.13 \mathrm{M})$ and cooled to $0{ }^{\circ} \mathrm{C}$. $\mathrm{NaBH}_{4}$ (167 mg, $4.41 \mathrm{mmol}, 3.7$ equiv.) was added, and the reaction was stirred for 30 min. The solution was quenched with saturated $\mathrm{NH}_{4} \mathrm{Cl}$ and allowed to come to ambient temperature. The material was extracted into EtOAc $(3 \times)$. The combined organic extracts were washed with brine, dried over $\mathrm{MgSO}_{4}$, filtered, and concentrated in vacuo. Purification by flash chromatography (silica, 30\% EtOAc/hexanes) yielded a diol (181 $\mathrm{mg}, 68 \%$ over 2 steps) as a colorless oil. The purified material (174 mg, $0.774 \mathrm{mmol})$ was dissolved in 2,2-dimethoxypropane $(7.74 \mathrm{~mL}, 0.1 \mathrm{M})$ and treated with $p-\mathrm{TsOH} \cdot \mathrm{H}_{2} \mathrm{O}$ monohydrate $(14.7 \mathrm{mg}, 0.774 \mathrm{mmol})$. The reaction was stirred for $36 \mathrm{~h}$ and quenched with water. The aqueous phase was extracted $3 \times$ into $\mathrm{CH}_{2} \mathrm{Cl}_{2}$. The combined organic extracts were washed with brine, dried over $\mathrm{MgSO}_{4}$, filtered, and concentrated in vacuo. Purification by flash chromatography (silica, 10\% EtOAc/hexanes) yielded dimethyl acetonide 10 (186 mg, 91\%) as a colorless oil. The oil (54.8 mg, $0.207 \mathrm{mmol})$ was then dissolved in $\mathrm{MeOH}(2.1 \mathrm{~mL}, 0.1 \mathrm{M})$ and treated with $10 \% \mathrm{Pd} / \mathrm{C}(10 \mathrm{mg}, 18 \%$ by wt.). The mixture was stirred under an $\mathrm{H}_{2}$ atmosphere for $24 \mathrm{~h}$ after which the material was filtered through a thin pad of silica gel and rinsed with EtOAc to remove the catalyst. Solvent removal under reduced pressure provided the primary alcohol (34.3 $\mathrm{mg}, 95 \%)$. A solution of the alcohol $(17.2 \mathrm{mg}, 0.0987 \mathrm{mmol})$ was prepared in $\mathrm{CH}_{2} \mathrm{Cl}_{2}(0.5 \mathrm{~mL}, 0.2$ M) and treated with pyridine ( $0.048 \mathrm{~mL}, 0.296 \mathrm{mmol}, 3.0$ equiv.), followed by DMAP (1.2 mg, $0.00987 \mathrm{mmol}, 0.1$ equiv.). The solution was cooled to $0{ }^{\circ} \mathrm{C}$ and acetic anhydride ( $0.013 \mathrm{~mL}, 0.148 \mathrm{mmol}, 1.5$ equiv.) was added. After $2 \mathrm{~h}$, the solution was quenched with saturated aqueous $\mathrm{NaHCO}_{3}$. The aqueous layer was extracted into $\mathrm{CH}_{2} \mathrm{Cl}_{2}$ (3x). The combined organic extracts were washed with brine, dried over $\mathrm{MgSO}_{4}$, filtered, and concentrated in vacuo. Purification by flash chromatography (silica, 10\% EtOAc/hexanes) yielded the acetylated dimethyl acetonide 11 (11 mg, $52 \%)$ as a colorless liquid. ${ }^{1} \mathrm{H}$ NMR $\left(400 \mathrm{MHz}, \mathrm{CDCl}_{3}\right) \delta 4.15(\mathrm{~m}, 2 \mathrm{H}), 3.67(\mathrm{dd}, J=$ 
5.0, $11.6 \mathrm{~Hz}, 1 \mathrm{H}), 3.54(\mathrm{ddd}, J=2.4,8.8,9.6 \mathrm{~Hz}, 1 \mathrm{H}), 3.49$ (dd, $J=11.2,11.6 \mathrm{~Hz}, 1 \mathrm{H}$ ), $2.03(\mathrm{~s}, 3 \mathrm{H}), 1.95(\mathrm{~m}, 1 \mathrm{H}), 1.66(\mathrm{~m}, 1 \mathrm{H}), 1.61$ (dddq, $J=5.0,6.8,9.6,11.2 \mathrm{~Hz}, 1 \mathrm{H})$, $1.39(\mathrm{~s}, 3 \mathrm{H}), 1.34(\mathrm{~s}, 3 \mathrm{H}), 0.74(\mathrm{~d}, J=6.8 \mathrm{~Hz}, 3 \mathrm{H})$.

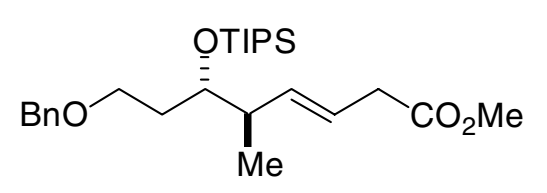

$(3 E, 5 R, 6 S)-8$-(Benzyloxy)-6-(triisopropylsilyloxy)-5methyloct-3-enoic acid methyl ester (13): A solution of homoallylic alcohol 9 (500 $\mathrm{mg}, 1.82 \mathrm{mmol})$ was prepared in $\mathrm{CH}_{2} \mathrm{Cl}_{2}(18.2 \mathrm{~mL}, 0.1 \mathrm{M})$ and cooled to $0{ }^{\circ} \mathrm{C}$. 2,6-lutidine $(0.55 \mathrm{~mL}, 4.74$ mmol, 2.6 equiv.) was added followed by TIPSOTf ( $0.64 \mathrm{~mL}, 2.37 \mathrm{mmol}, 1.3$ equiv). The reaction was stirred $24 \mathrm{~h}$ and quenched with saturated aqueous $\mathrm{NH}_{4} \mathrm{Cl}(20 \mathrm{~mL})$. The phases were separated and the aqueous layer was extracted into $\mathrm{CH}_{2} \mathrm{Cl}_{2}(3 \times)$. The combined organic layers were dried with $\mathrm{MgSO}_{4}$, filtered, and concentrated in vacuo. Purification by flash chromatography (silica, 3\% EtOAc/hexanes) gave TIPS ether $\mathbf{1 2}$ as a colorless oil (813 mg, 99\%). ${ }^{1} \mathrm{H}$ NMR $\left(270 \mathrm{MHz}, \mathrm{CDCl}_{3}\right) \delta$ 7.26-7.35 (m, 5H), 5.48$5.51(\mathrm{~m}, 2 \mathrm{H}), 4.45(\mathrm{ABq}, J=16.6 \mathrm{~Hz}, 2 \mathrm{H}), 3.91(\mathrm{~m}, 1 \mathrm{H}), 3.65(\mathrm{~s}, 3 \mathrm{H}), 3.50(\mathrm{t}, J=6.8$ $\mathrm{Hz}, 2 \mathrm{H}), 3.01(\mathrm{~d}, J=4.4 \mathrm{~Hz}, 2 \mathrm{H}), 2.30-2.40(\mathrm{~m}, 1 \mathrm{H}), 1.70(\mathrm{dt}, J=6.4,6.8 \mathrm{~Hz}, 2 \mathrm{H}), 1.03$ (m, 21H), 1.00 (par obsc m, 3H); ${ }^{13} \mathrm{C}$ NMR $\left(67.9 \mathrm{MHz}, \mathrm{CDCl}_{3}\right) \delta 172.4,138.5,136.4$, 128.2, 127.6, 127.4, 121.8, 73.1, 72.8, 67.3, 51.6, 42.2, 38.0, 33.5, 18.2, 14.6, 12.9; IR (neat) $v_{\max } 2944,2892,2866,1743,1456,1165,1103$; HRMS (CI, NH $\left.\mathrm{N}_{3}\right), \mathrm{m} / z$ calculated for $\mathrm{C}_{26} \mathrm{H}_{45} \mathrm{O}_{4} \mathrm{Si}[\mathrm{M}+\mathrm{H}]^{+}$449.3087, found: 449.3062; $[\alpha]^{23}{ }_{\mathrm{D}}=+9^{\circ}\left(c 3.5, \mathrm{CHCl}_{3}\right)$.

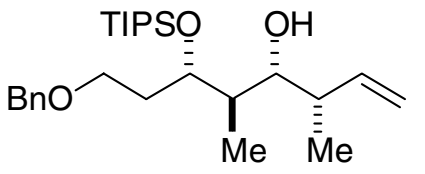

$(3 S, 4 R, 5 R, 6 S)-8$-(Benzyloxy)-3,5-dimethyl-6(triisopropylsilyloxy)-oct-1-en-4-ol (5): TIPS ether $\mathbf{1 2}$ $(1.26 \mathrm{~g}, 2.79 \mathrm{mmol})$ and pyridine $(0.23 \mathrm{~mL}, 2.79 \mathrm{mmol}, 1.0$ equiv.) were dissolved in $\mathrm{MeOH}(31 \mathrm{~mL}, 0.09 \mathrm{M})$ and cooled to $-78{ }^{\circ} \mathrm{C}$. Ozone was bubbled through the mixture until the starting material was consumed as determined by TLC analysis (20\% EtOAc/hexanes). The reaction was quenched with $\mathrm{Me}_{2} \mathrm{~S}(3.46 \mathrm{~mL}$, $55.8 \mathrm{mmol}, 20$ equiv.) and allowed to warm to ambient temperature over $12 \mathrm{~h}$. The solution was concentrated directly in vасио and the crude residue was diluted with EtOAc. This material was washed with $\mathrm{H}_{2} \mathrm{O}(3 \times)$ and with brine $(1 \times)$. The organic 
layer was dried with $\mathrm{MgSO}_{4}$, filtered and concentrated to yield the crude aldehyde, which was used immediately without further purification. A solution of $(S, S)$ diisopropyltartrate-modified $(Z)$-crotylboronate $\mathbf{8}^{4}(8.25 \mathrm{~mL}$ of $0.44 \mathrm{M}$ solution in toluene, $3.63 \mathrm{mmol}, 1.3$ equiv.) and $4 \AA$ molecular sieves (190 mg) was cooled to -78 ${ }^{\circ} \mathrm{C}$. To this solution was added the crude $\alpha$-methyl aldehyde $(1.06 \mathrm{~g}, 2.79 \mathrm{mmol}$ theoretical yield) in toluene $(7.35 \mathrm{~mL}, 0.18 \mathrm{M}$ total vol.) dropwise over $20 \mathrm{~min}$. The mixture was stirred at $-78{ }^{\circ} \mathrm{C}$ for $4 \mathrm{~h}$, quenched with $1 \mathrm{M} \mathrm{NaOH}(7 \mathrm{~mL})$, stirred at $0{ }^{\circ} \mathrm{C}$ for $1 \mathrm{~h}$, allowed to come to ambient temperature, and stirred an additional $1 \mathrm{~h}$. The phases were separated and the aqueous layer was extracted with $\mathrm{Et}_{2} \mathrm{O}(3 \times)$. The combined organic layers were washed with brine, dried over $\mathrm{MgSO}_{4}$, filtered, and concentrated in vacuo. Purification by flash chromatography (silica, 2.5\% EtOAc/hexanes) yielded 5 (780 mg, 64\% over two steps) as a colorless oil. ${ }^{1} \mathrm{H}$ NMR $\left(300 \mathrm{MHz}, \mathrm{CDCl}_{3}\right) \delta$ 7.29-7.31 (m, 5H), $5.90(\mathrm{ddd}, J=6.0,11.0,17.0 \mathrm{~Hz}, 1 \mathrm{H}), 5.08(\mathrm{~d}$, $J=11.0 \mathrm{~Hz}, 1 \mathrm{H}), 5.06(\mathrm{~d}, J=17.0 \mathrm{~Hz}, 1 \mathrm{H}), 4.48(\mathrm{ABq}, J=19.5 \mathrm{~Hz}, 2 \mathrm{H}), 4.30(\mathrm{~m}, 1 \mathrm{H})$, 3.55-3.61 (m, 1H), 3.36 (dd, $J=2.1,9.9 \mathrm{~Hz}, 1 \mathrm{H}), 2.37(\mathrm{~m}, 1 \mathrm{H}), 1.92(\mathrm{br} \mathrm{s}, 1 \mathrm{H}), 1.88$ (m, 2H), $1.65(\mathrm{~m}, 1 \mathrm{H}), 1.23(\mathrm{~m}, 2 \mathrm{H}), 1.03(\mathrm{~m}, 21 \mathrm{H}), 0.94(\mathrm{~d}, J=0.7 \mathrm{~Hz}, 3 \mathrm{H}), 0.80(\mathrm{~d}, J$ $=0.7 \mathrm{~Hz}, 3 \mathrm{H}) ;{ }^{13} \mathrm{C} \mathrm{NMR}\left(67.9 \mathrm{MHz}, \mathrm{CDCl}_{3}\right) \delta 142.6,138.2,128.2,127.7,127.5,114.4$, 75.3, 73.0, 70.5, 68.0, 41.6, 39.1, 32.5, 18.1, 12.7, 10.4, 10.3; IR (neat) $v_{\max } 3456,2962$, 2943, 2866, 1463, 1106, 1063, 676; HRMS (CI, $\left.\mathrm{NH}_{3}\right), \mathrm{m} / z$ calculated for $\mathrm{C}_{26} \mathrm{H}_{47} \mathrm{O}_{3} \mathrm{Si}$ $[\mathrm{M}+\mathrm{H}]^{+} 435.3294$, found: $435.3314 ;[\alpha]^{23}{ }_{\mathrm{D}}=-15^{\circ}\left(c 10.2, \mathrm{CHCl}_{3}\right)$.

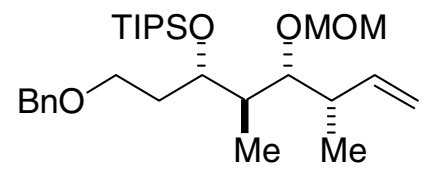

(3S,4R,5R,6S)-8-(Benzyloxy)-3,5-dimethyl-4methoxymethoxy-6-(triisopropylsilyloxy)-oct-1-ene (13): A solution of homoallylic alcohol 5 (406 $\mathrm{mg}, 0.93 \mathrm{mmol})$ and DIPEA ( $0.98 \mathrm{~mL}, 5.6 \mathrm{mmol}$, 6.0 equiv.) in $\mathrm{CH}_{2} \mathrm{Cl}_{2}(3.2 \mathrm{~mL}, 0.3 \mathrm{M})$ was cooled to 0 ${ }^{\circ} \mathrm{C}$ and treated with DMAP (35 mg, $0.28 \mathrm{mmol}, 0.3$ equiv.) followed by chloromethyl methyl ether $(0.285 \mathrm{~mL}, 3.73 \mathrm{mmol}, 4.0$ equiv.). The reaction was allowed to come to ambient temperature over $18 \mathrm{~h}$ and quenched with saturated $\mathrm{NaHCO}_{3}(5 \mathrm{~mL})$. The aqueous phase was extracted into $\mathrm{CH}_{2} \mathrm{Cl}_{2}(3 \times)$. The combined organic extracts were

\footnotetext{
${ }^{4}$ For preparation and review of tartrate modified crotylboronates, see: Roush, W. R.; Kaori, A.; Powers, D. B.; Palkowitz, A. D.; Halterman, R. L. J. Am. Chem. Soc. 1990, 112, 6339-6348.
} 
washed with brine, dried over $\mathrm{MgSO}_{4}$, filtered, and concentrated in vacuo. Purification by flash chromatography (silica, 2.5\% EtOAc/hexanes) afforded $\mathbf{1 3}$ (375 $\mathrm{mg}, 84 \%$ ) as a colorless oil. ${ }^{1} \mathrm{H}$ NMR $\left(270 \mathrm{MHz}, \mathrm{CDCl}_{3}\right) \delta$ 7.25-7.31 (m, 5H), 5.91 (ddd, $J=6.6$, $10.5,17.1 \mathrm{~Hz}, 1 \mathrm{H}), 5.00(\mathrm{~d}, J=17.1 \mathrm{~Hz}, 1 \mathrm{H}), 4.97(\mathrm{~d}, J=10.5 \mathrm{~Hz}, 1 \mathrm{H}), 4.50(\mathrm{ABq}, J=$ 8.6, $2 \mathrm{H}), 4.46(\mathrm{ABq}, J=15.2 \mathrm{~Hz}, 2 \mathrm{H}), 4.27(\mathrm{~m}, 1 \mathrm{H}), 3.54-3.62(\mathrm{~m}, 2 \mathrm{H}), 3.25(\mathrm{~s}, 3 \mathrm{H})$, $3.22(\mathrm{dd}, J=2.0,9.9 \mathrm{~Hz}, 1 \mathrm{H}), 2.37(\mathrm{~m}, 1 \mathrm{H}), 1.95(\mathrm{~m}, 1 \mathrm{H}), 1.77(\mathrm{~m}, 1 \mathrm{H}), 1.60(\mathrm{~m}, 1 \mathrm{H})$, $1.03(\mathrm{~m}, 21 \mathrm{H}), 0.96(\mathrm{~d}, J=7.9 \mathrm{~Hz}, 3 \mathrm{H}), 0.82(\mathrm{~d}, J=6.6 \mathrm{~Hz}, 3 \mathrm{H}) .{ }^{13} \mathrm{C} \mathrm{NMR}(67.9 \mathrm{MHz}$, $\left.\mathrm{CDCl}_{3}\right) \delta 142.8,138.5,128.1,127.6,127.3,113.4,98.2,72.9,69.0,68.0,56.0,42.3$, 39.4, 31.6, 18.1, 12.7, 11.9, 10.2; IR (neat) $v_{\max }$ 2964, 2943, 2891, 2867, 1464, 1097, 1034, 883; HRMS (CI, $\left.\mathrm{NH}_{3}\right), \mathrm{m} / z$ calculated for $\mathrm{C}_{28} \mathrm{H}_{50} \mathrm{O}_{4} \mathrm{Si}[\mathrm{M}+\mathrm{H}]^{+} 479.3557$, found: $479.3483 ;[\alpha]^{23}{ }_{\mathrm{D}}=-31^{\circ}\left(c 5.7, \mathrm{CHCl}_{3}\right)$.

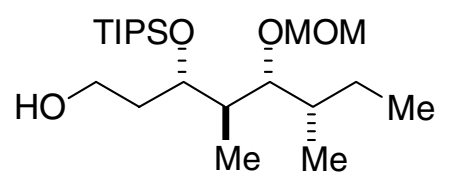

$(3 S, 4 R, 5 R, 6 S)$-4,6-Dimethyl-5-methoxymethoxy-3(triisopropylsilyloxy)-octan-1-ol (14): To a solution of MOM ether 13 (366 mg, $0.76 \mathrm{mmol}$ ) dissolved in $\mathrm{MeOH}$ (8 $\mathrm{mL}, 0.1 \mathrm{M}$ ) was added $10 \%$ palladium on activated carbon $(110 \mathrm{mg}, 30 \%$ by wt.). The mixture was kept under positive pressure of $\mathrm{H}_{2}$ and stirred $24 \mathrm{~h}$. The material was then passed through a thin pad of silica gel to remove the solids. Concentration in vacuo yielded a residue that was then subjected to flash chromatography (silica, 10\% EtOAc/hexanes) which afforded $14(258 \mathrm{mg}, 86 \%)$ as a colorless oil. ${ }^{1} \mathrm{H}$ NMR (270 $\left.\mathrm{MHz}, \mathrm{CDCl}_{3}\right) \delta 4.59(\mathrm{ABq}, J=10.3 \mathrm{~Hz}, 2 \mathrm{H}), 4.40(\mathrm{dt}, J=3.4,8.8 \mathrm{~Hz}, 1 \mathrm{H}), 3.77(\mathrm{t}, J=$ $5.5 \mathrm{~Hz}, 2 \mathrm{H}), 3.36$ (s, 3H), 3.14 (dd, $J=1.5,10.3 \mathrm{~Hz}, 1 \mathrm{H}), 1.95-2.05(\mathrm{~m}, 1 \mathrm{H}), 1.60-1.68$ (m, 1H), 1.15-1.55 (par obsc m, 5H), $1.08(\mathrm{~m}, 21 \mathrm{H}), 0.90(\mathrm{t}, J=7.3,3 \mathrm{H}), 0.82(\mathrm{~d}, J=$ 6.8, 3H), $0.81(\mathrm{~d}, J=6.8,3 \mathrm{H}) ;{ }^{13} \mathrm{C}$ NMR $\left(67.9 \mathrm{MHz}, \mathrm{CDCl}_{3}\right) \delta$ 99.2, 85.9, 70.6, 60.7, 56.1, 41.8, 37.2, 33.7, 27.4, 18.2, 12.9, 12.3, 12.2, 10.3; IR (neat) $v_{\max } 3440,3963$, 2943, 2892, 2867, 1464, 1382, 1140, 1088, 1041, 883; HRMS (CI, $\mathrm{NH}_{3}$ ), $m / z$ calculated for $\mathrm{C}_{21} \mathrm{H}_{47} \mathrm{O}_{4} \mathrm{Si}[\mathrm{M}+\mathrm{H}]^{+} 391.3244$, found: $391.3243 ;[\alpha]^{23}{ }_{\mathrm{D}}=-44^{\circ}\left(c\right.$ 2.0, $\left.\mathrm{CHCl}_{3}\right)$.

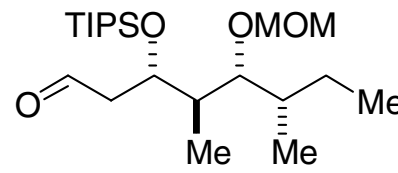

$(3 S, 4 R, 5 R, 6 S)-4,6-D i m e t h y l-5-m e t h o x y m e t h o x y-3-$ (triisopropylsilyloxy)-octanal (15): To a solution of oxalyl 
chloride (0.154 mL, $1.77 \mathrm{mmol}, 2.3$ equiv.) in $\mathrm{CH}_{2} \mathrm{Cl}_{2}(5.3 \mathrm{~mL})$ at $-78{ }^{\circ} \mathrm{C}$ was added DMSO (0.24 mL, $3.38 \mathrm{mmol}, 4.4$ equiv.) dropwise. The solution was stirred for $15 \mathrm{~min}$ and 14 (300 mg, $0.769 \mathrm{mmol})$ in $\mathrm{CH}_{2} \mathrm{Cl}_{2}$ (3.2 mL, including rinses, $0.9 \mathrm{M}$ total volume) was added slowly. The solution was stirred $15 \mathrm{~min}$ and $\mathrm{Et}_{3} \mathrm{~N}(1.06 \mathrm{~mL}, 7.69 \mathrm{mmol})$ was added. After $15 \mathrm{~min}$, the cold bath was removed and the reaction was stirred an additional $1 \mathrm{~h}$ at ambient temperature. The solution was quenched with water $(10 \mathrm{~mL})$. The aqueous phase was extracted into $\mathrm{CH}_{2} \mathrm{Cl}_{2}(3 \times)$. The combined organic extracts were washed with brine, dried over $\mathrm{MgSO}_{4}$, filtered, and concentrated in vacuo. The yellow residue was purified by flash chromatography (silica, 5\% EtOAc/hexanes) to yield 15 (292 mg, 98\%) as a pale yellow oil. ${ }^{1} \mathrm{H} \mathrm{NMR}\left(270 \mathrm{MHz}, \mathrm{CDCl}_{3}\right) \delta 9.86(\mathrm{~m}$, $1 \mathrm{H}), 4.81(\mathrm{dt}, J=3.4,7.8 \mathrm{~Hz}, 1 \mathrm{H}), 4.56(\mathrm{ABq}, J=8.8 \mathrm{~Hz}, 2 \mathrm{H}), 3.34$ (s, 3H), 3.08 (dd, $J$ $=1.5,9.8 \mathrm{~Hz}, 1 \mathrm{H}), 2.40-2.50(\mathrm{~m}, 2 \mathrm{H}), 2.01-2.04(\mathrm{~m}, 1 \mathrm{H}), 1.25-1.60$ (par obsc m, 3H), $1.04(\mathrm{~m}, 21 \mathrm{H}), 0.91(\mathrm{t}, J=7.3 \mathrm{~Hz}, 3 \mathrm{H}), 0.83(\mathrm{~d}, J=7.3 \mathrm{~Hz}, 3 \mathrm{H}), 0.83(\mathrm{~d}, J=7.3 \mathrm{~Hz}$, $3 \mathrm{H}) ;{ }^{13} \mathrm{C} \mathrm{NMR}\left(67.9 \mathrm{MHz}, \mathrm{CDCl}_{3}\right) \delta 203.1,98.7,85.1,68.1,56.1,45.8,41.8,37.3$, 27.2, 18.0, 12.6, 12.5, 12.3, 10.5; IR (neat) $v_{\max } 2943,2964,2868,1730,1464,1383$, 1141, 1094, 1038, 883; HRMS (CI, $\left.\mathrm{NH}_{3}\right), m / z$ calculated for $\mathrm{C}_{21} \mathrm{H}_{45} \mathrm{O}_{4} \mathrm{Si}[\mathrm{M}+\mathrm{H}]^{+}$ 389.3087, found: $389.3194 ;[\alpha]^{23}=-35^{\circ}\left(c 4.5, \mathrm{CHCl}_{3}\right)$.

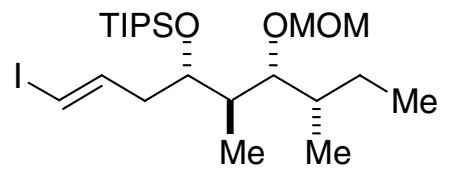

$(1 E, 4 S, 5 R, 6 R, 7 S)-5,7-D i m e t h y l-1-i o d o-6-$ methoxymethoxy-4-(triisopropylsilyloxy)-oct-1-ene (4): A stirred suspension of $\mathrm{CrCl}_{2}(33.1 \mathrm{mg}, 0.270 \mathrm{mmol}, 8.0$ equiv.) in 6:1 dioxane/THF $(0.15 \mathrm{~mL})$ was prepared and cooled to $0{ }^{\circ} \mathrm{C}$. To this suspension was added a solution of aldehyde 15 (13.1 $\mathrm{mg}, 0.0337 \mathrm{mmol})$ and $\mathrm{CHI}_{3}$ (39.8 mg, $0.101 \mathrm{mmol}, 3.0$ equiv.) in $6: 1$ dioxane/THF $(0.75 \mathrm{~mL}$, including rinses, 0.04 $\mathrm{M}$ final total volume). The reaction was protected from light and allowed to come to ambient temperature over $20 \mathrm{~h}$. The green solution was quenched with water. The aqueous layer was extracted with EtOAc $(3 \times)$. The combined organic extracts were washed with saturated aqueous $\mathrm{Na}_{2} \mathrm{~S}_{2} \mathrm{O}_{3}$ followed by brine. The material was then dried over $\mathrm{MgSO}_{4}$, filtered, and concentrated. Purification by flash chromatography (silica, $1 \%$ EtOAc/hexanes) yielded $4(14.3 \mathrm{mg}, 83 \%)$ as a pale yellow oil. ${ }^{1} \mathrm{H}$ NMR (270 
$\left.\mathrm{MHz}, \mathrm{CDCl}_{3}\right) \delta 6.60(\mathrm{~m}, 1 \mathrm{H}), 6.00(\mathrm{~d}, J=14.2 \mathrm{~Hz}, 1 \mathrm{H}), 4.56(\mathrm{ABq}, J=11.7 \mathrm{~Hz}, 2 \mathrm{H})$, $4.21(\mathrm{~m}, 1 \mathrm{H}), 3.34(\mathrm{~s}, 3 \mathrm{H}), 3.16(\mathrm{~m}, 1 \mathrm{H}), 1.95-2.26(\mathrm{~m}, 3 \mathrm{H}), 1.25-1.50(\mathrm{~m}, 3 \mathrm{H}), 1.05$ (m, 21H), 0.89 (t, $J=3.9 \mathrm{~Hz}, 3 \mathrm{H}), 0.81(\mathrm{~d}, J=6.3 \mathrm{~Hz}, 3 \mathrm{H}), 0.79$ (d, $J=6.8 \mathrm{~Hz}, 3 \mathrm{H})$; ${ }^{13} \mathrm{C}$ NMR $\left(67.9 \mathrm{MHz}, \mathrm{CDCl}_{3}\right) \delta 145.2,98.7,85.0,76.0,71.4,56.2,42.1,38.3,37.3$, 27.4, 18.2, 12.7, 12.4, 12.3, 10.4; IR (neat) $v_{\max }$ 2943, 2963, 2867, 1464, 1381, 1040, 1088, 1038, 883; HRMS (CI, $\mathrm{NH}_{3}$ ), $\mathrm{m} / z$ calculated for $\mathrm{C}_{22} \mathrm{H}_{46} \mathrm{IO}_{3} \mathrm{Si}[\mathrm{M}+\mathrm{H}]^{+}$513.2261, found: $513.2230 ;[\alpha]^{23}{ }_{D}=-67^{\circ}\left(c 2.4, \mathrm{CHCl}_{3}\right)$.

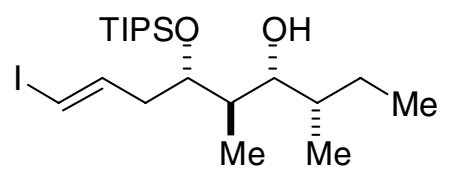

$(1 E, 4 S, 5 R, 6 R, 7 S)-5,7-D i m e t h y l-1-i o d o-4-$ (triisopropylsilyloxy)-oct-1-en-6-ol (16): A sample of vinyl iodide 4 (163mg, $0.318 \mathrm{mmol}$ ) was dissolved in $\mathrm{CH}_{2} \mathrm{Cl}_{2}(12 \mathrm{~mL}, 0.03 \mathrm{M})$ and cooled to $-78^{\circ} \mathrm{C}$. To this solution was added $\mathrm{BCl}_{3}(0.72$ $\mathrm{mL}$ of a $1 \mathrm{M}$ solution in hexane, 2.3 equiv.). The reaction was protected from light, stirred for $2 \mathrm{~h}$, and while still at $-78{ }^{\circ} \mathrm{C}$, quenched with $\mathrm{MeOH}(2.5 \mathrm{~mL})$ followed by $50 \%$ saturated $\mathrm{NaHCO}_{3}(5 \mathrm{~mL})$. The cold bath was removed and the reaction was allowed to come to ambient temperature. The aqueous layer was extracted into $\mathrm{CH}_{2} \mathrm{Cl}_{2}$ (3×). The combined organic extracts were washed with brine, dried over $\mathrm{MgSO}_{4}$, filtered, and concentrated. Purification by flash chromatography (silica, 2.5\% EtOAc/hexanes) afforded alcohol 16 (112 mg, 75\%) as a pale yellow oil. ${ }^{1} \mathrm{H}$ NMR (270 $\left.\mathrm{MHz}, \mathrm{CDCl}_{3}\right) \delta 6.58(\mathrm{~m}, 1 \mathrm{H}), 6.03(\mathrm{~d}, J=14.7 \mathrm{~Hz}, 1 \mathrm{H}), 4.18(\mathrm{~m}, 1 \mathrm{H}), 3.39(\mathrm{~m}, 1 \mathrm{H})$, 2.12-2.36 (m, 2H), $1.85(\mathrm{~m}, 1 \mathrm{H}), 1.60(\mathrm{br} \mathrm{s}, 1 \mathrm{H}), 1.30-1.50(\mathrm{~m}, 3 \mathrm{H}), 1.06(\mathrm{~m}, 21 \mathrm{H})$, $0.90(\mathrm{~m}, 3 \mathrm{H}), 0.80(\mathrm{~d}, J=6.8 \mathrm{~Hz}, 3 \mathrm{H}), 0.78(\mathrm{~d}, J=6.8 \mathrm{~Hz}, 3 \mathrm{H}) ;{ }^{13} \mathrm{C} \mathrm{NMR}(67.9 \mathrm{MHz}$, $\left.\mathrm{CDCl}_{3}\right) \delta 144.2,76.3,75.6,73.3,41.6,39.9,36.5,27.2,18.2,18.1,12.7,12.1,11.4 ; \mathrm{IR}$ (neat) $v_{\max } 3510,2962,2942,2866,1463,1382,1086,1065,677$; HRMS (CI, $\left.\mathrm{NH}_{3}\right), m / z$ calculated for $\mathrm{C}_{20} \mathrm{H}_{42} \mathrm{IO}_{2} \mathrm{Si}[\mathrm{M}+\mathrm{H}]^{+} 469.1999$, found: 469.2052; $[\alpha]^{23}{ }_{\mathrm{D}}=-6^{\circ}(c 0.76$, $\left.\mathrm{CHCl}_{3}\right)$.

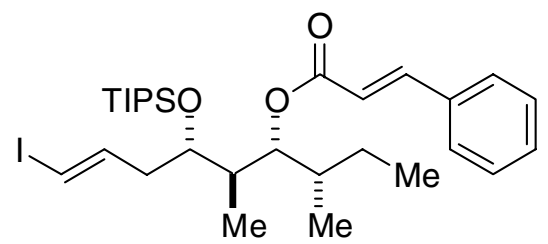

(1E,4S,5R,6R,7S)-5,7-Dimethyl-1-iodo-4(triisopropylsilyloxy)-6-[(2E)-3-phenylacryloxy] oct-1-ene (17): A solution of secondary alcohol 16 
(14.9 mg, $0.0318 \mathrm{mmol})$ in $\mathrm{CH}_{2} \mathrm{Cl}_{2}(0.3 \mathrm{~mL}, 0.1 \mathrm{M})$ was treated with $\mathrm{Et}_{3} \mathrm{~N}(0.04 \mathrm{~mL}$, $0.254 \mathrm{mmol}, 8.0$ equiv.) and DMAP (1.2 mg, $0.0095 \mathrm{mmol}, 0.3$ equiv.) and cooled to 0 ${ }^{\circ} \mathrm{C}$. The solution was treated with a solution of $(E)$-cinnamoyl chloride $(21.2 \mathrm{mg}, 0.127$ mmol, 4.0 equiv.) dissolved in $\mathrm{CH}_{2} \mathrm{Cl}_{2}(0.70 \mathrm{~mL}, 0.03 \mathrm{M}$ total vol.). The reaction was protected from light and allowed to come to ambient temperature $48 \mathrm{~h}$. The reaction was then quenched with water. The aqueous phase was extracted into $\mathrm{CH}_{2} \mathrm{Cl}_{2}(3 \times)$. The combined organic extracts were washed with brine, dried over $\mathrm{MgSO}_{4}$, filtered and concentrated under reduced pressure. Purification by flash chromatography (silica, 1\% EtOAc/hexanes) yielded 17 (17.6 mg, 92\%) as a yellow oil. ${ }^{1} \mathrm{H}$ NMR (270 MHz, $\left.\mathrm{CDCl}_{3}\right) \delta 7.64(\mathrm{~d}, J=16.1 \mathrm{~Hz}, 1 \mathrm{H}), 7.35-7.53(\mathrm{~m}, 5 \mathrm{H}), 6.54(\mathrm{~m}, 1 \mathrm{H}), 6.38(\mathrm{~d}, 16.1 \mathrm{~Hz}$ $1 \mathrm{H}), 6.00(\mathrm{~d}, J=14.2 \mathrm{~Hz}, 1 \mathrm{H}), 4.92(\mathrm{dd}, J=1.5,10.7 \mathrm{~Hz}, 1 \mathrm{H}), 3.83$ (m, 1H), 2.25-2.35 (m, 1H), 2.00-2.20 (m, 1H), $1.65(\mathrm{~m}, 1 \mathrm{H}), 1.10-1.35(\mathrm{~m}, 3 \mathrm{H}), 0.90$ (obsc m, 9H); ${ }^{13} \mathrm{C}$ NMR (67.9 MHz, $\left.\mathrm{CDCl}_{3}\right) \delta 166.5,145.0,144.8,134.4,130.3,128.9,128.1,117.9$, 76.7, 76.5, 71.7, 41.5, 38.1, 36.2, 26.9, 18.1, 12.7, 12.3, 12.0, 9.6; IR (neat) $v_{\max } 2964$, 2942, 2866, 1709, 1639, 1173, 1089, 766; HRMS (CI, $\mathrm{NH}_{3}$ ), $\mathrm{m} / \mathrm{z}$ calculated for $\mathrm{C}_{29} \mathrm{H}_{48} \mathrm{IO}_{3} \mathrm{Si}[\mathrm{M}+\mathrm{H}]^{+}$599.2417, found: 599.2365; $[\alpha]^{23}{ }_{\mathrm{D}}=-55^{\circ}\left(c 0.88, \mathrm{CHCl}_{3}\right)$.

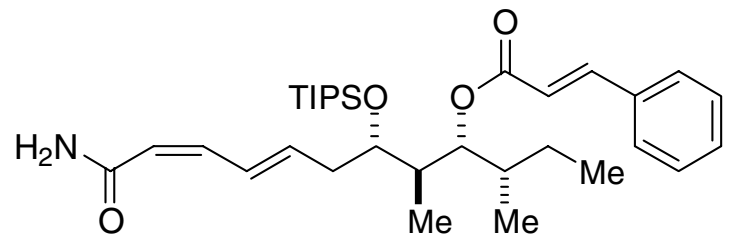

$(2 Z, 4 E, 7 S, 8 R, 9 R, 10 S)-8,10-D i m e t h y l-7-$ (triisopropylsilyloxy)-9-[(2E)-3phenylacryloxy]-dodec-2,4-dienamide (18): Cinnamoyl ester 17 (15.8 mg, $0.0264 \mathrm{mmol})$ was treated with a solution of vinyl stannane $\mathbf{3}^{5}(8.7 \mathrm{mg}, 0.0369 \mathrm{mmol}$, 1.4 equiv.) in $\mathrm{DMF}(0.64 \mathrm{~mL}, 0.04 \mathrm{M}$ based on 17). Dichloro(bis)acetonitrile palladium(II) $(0.5 \mathrm{mg}, 0.0013 \mathrm{mmol}, 0.05$ equiv.) was then added. The reaction was protected from light and stirred at ambient temperature $18 \mathrm{~h}$, at which time additional amounts of stannane ( $8.7 \mathrm{mg}, 0.0369 \mathrm{mmol}, 1.4$ equiv.) and dichloro(bis)acetonitrile palladium(II) ( $0.5 \mathrm{mg}, 0.0013 \mathrm{mmol}, 0.05$ equiv.) were added. The reaction was stirred an additional $24 \mathrm{~h}$ before it was quenched with water. The mixture was diluted with

\footnotetext{
${ }^{5}$ Ermolenko, M. S. Tetrahedron Lett. 1996, 37, 6711-6712; the ethyl ester was reported in Stille. J. K.; Groh, B. L. J. Am. Chem. Soc. 1987, 109, 813-817; while the amidation was performed using conditions detailed in Basha, A.; Lipton, M.; Weinreb, S. M.; Tetrahedron Lett. 1977, 48, 4171-4174.
} 
EtOAc and washed with water $(3 \times)$. The combined aqueous layers were reextracted with EtOAc $(1 \times)$ and the combined organic layers were washed with brine, dried over $\mathrm{MgSO}_{4}$, filtered, and concentrated in vacuo. Purification by flash chromatography (silica, 60\% EtOAc/hexanes) yielded diene $18(10.8 \mathrm{mg}, 76 \%)$ as a faint yellow oil. ${ }^{1} \mathrm{H}$ NMR (270 MHz, $\left.\mathrm{CDCl}_{3}\right) \delta 7.65(\mathrm{~d}, J=16.1 \mathrm{~Hz}, 1 \mathrm{H}), 7.50(\mathrm{~m}, 2 \mathrm{H}), 7.40(\mathrm{~m}, 3 \mathrm{H}), 7.23$ (par obsc m, 1H), 6.41 (par obsc m, 1H), 6.40 (d, $J=16.1 \mathrm{~Hz}, 1 \mathrm{H}), 6.05(\mathrm{dt}, J=7.2$, $14.4 \mathrm{~Hz}, 1 \mathrm{H}), 5.51$ (d, $J=11.0 \mathrm{~Hz}, 1 \mathrm{H}), 4.95$ (dd, $J=1.2,11.6 \mathrm{~Hz}, 1 \mathrm{H}), 3.90(\mathrm{~m}, 1 \mathrm{H})$, $2.45(\mathrm{~m}, 1 \mathrm{H}), 2.27(\mathrm{~m}, 1 \mathrm{H}), 2.15(\mathrm{~m}, 1 \mathrm{H}), 1.60(\mathrm{~m}, 2 \mathrm{H}), 1.10-1.25(\mathrm{~m}, 2 \mathrm{H}), 0.99(\mathrm{~m}$, 21H), 0.90 (ovlp m, 9H); ${ }^{13} \mathrm{C}$ NMR (67.9 MHz, $\mathrm{CDCl}_{3}$ ) $\delta$ 168.1, 166.5, 144.9, 142.3, $142.0,134.4,130.3,128.9,128.2$, 128.1, 118.0, 117.6, 77.2, 72.0, 41.8, 36.2, 35.7, 26.9, 18.1, 12.6, 12.3, 12.0, 9.8; IR (neat) $v_{\max } 3347,3201,2959,2956,1709,1662,1637$, 1463, 1082, 990; HRMS (CI, $\left.\mathrm{NH}_{3}\right), \mathrm{m} / z$ calculated for $\mathrm{C}_{32} \mathrm{H}_{52} \mathrm{NO}_{4} \mathrm{Si}[\mathrm{M}+\mathrm{H}]^{+} 542.3666$, found: $542.3727 ;[\alpha]^{23}{ }_{\mathrm{D}}=-119^{\circ}\left(c 0.15, \mathrm{CHCl}_{3}\right)$.

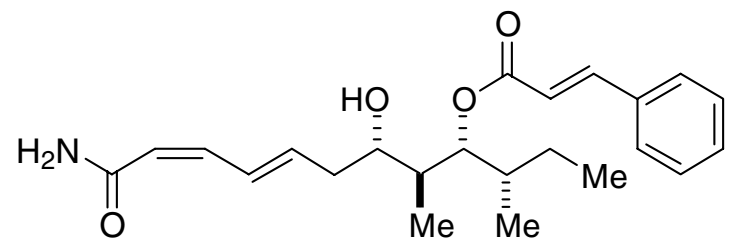

Basiliskamide A (1a): Diene 18 (2.6 mg, $0.00480 \mathrm{mmol}$ ) was dissolved in THF $(0.20 \mathrm{~mL}, 0.024 \mathrm{M})$ and placed in a plastic vial. The solution was treated with $70 \%$

$\mathrm{HF} /$ pyridine $(0.20 \mathrm{~mL}, 1: 1 \mathrm{vol}$. equiv. vs. THF) and stirred $12 \mathrm{~h}$. The solution was quenched with $\mathrm{H}_{2} \mathrm{O}$, followed by saturated $\mathrm{NaHCO}_{3}$. The aqueous phase was extracted with EtOAc $(3 \times)$. The combined organic extracts were washed with brine, dried over $\mathrm{MgSO}_{4}$, filtered, and concentrated in vacuo. Purification by flash chromatography (silica, 75\% EtOAc/ $\mathrm{CH}_{2} \mathrm{Cl}_{2}$ ) afforded basiliskamide A 1a (1.4 mg, 75\%). ${ }^{1} \mathrm{H} \mathrm{NMR}$ (400 MHz, DMSO-d $\left.d^{6}\right) \delta 7.72(\mathrm{~m}, 2 \mathrm{H}), 7.66(\mathrm{~d}, J=15.9 \mathrm{~Hz}, 1 \mathrm{H}), 7.40(\mathrm{~m}, 3 \mathrm{H}), 7.40$ (par obsc m, 1H), $7.36(\mathrm{~s}, 1 \mathrm{H}), 6.88(\mathrm{~s}, 1 \mathrm{H}), 6.63$ (d, $J=15.9 \mathrm{~Hz}, 1 \mathrm{H}), 6.30$ (dt, $J=$ 11.3, 11.6 Hz, 1H), 5.90 (dt, $J=7.3,14.6 \mathrm{~Hz}, 1 \mathrm{H}), 5.55$ (d, $J=11.6 \mathrm{~Hz}, 1 \mathrm{H}), 4.92$ (m, $1 \mathrm{H}), 4.61(\mathrm{~d}, J=5.2 \mathrm{~Hz}, 1 \mathrm{H}), 3.47$ (m, 1H), 2.28 (m, 1H), 2.01 (m, 1H), $1.99(\mathrm{~m}, 1 \mathrm{H})$, $1.68(\mathrm{~m}, 1 \mathrm{H}), 1.25(\mathrm{~m}, 1 \mathrm{H}), 1.11(\mathrm{~m}, 1 \mathrm{H}), 0.90(\mathrm{~d}, J=7.2 \mathrm{~Hz}, 3 \mathrm{H}), 0.87$ (t, $J=7.6 \mathrm{~Hz}$, $3 \mathrm{H}), 0.83(\mathrm{~d}, J=6.8 \mathrm{~Hz}, 3 \mathrm{H}) ;{ }^{13} \mathrm{C}$ NMR $\left(75.0 \mathrm{MHz}, \mathrm{DMSO}-d_{6}\right) \delta 168.3,166.8,145.4$, $141.3,134.7,131.1,129.6,129.1,129.0,120.0,118.6,76.8,70.2$, 41.3, 36.0, 35.1, 27.0, 
13.3, 12.2, 10.5; IR (neat) $v_{\max } 3352,3204,2956,2925,2853,1706,1701,1669,1636$, 1511, 1248; HRMS (CI, $\mathrm{NH}_{3}$ ) $\mathrm{m} / z$ calculated for $\mathrm{C}_{23} \mathrm{H}_{32} \mathrm{NO}_{4}[\mathrm{M}+\mathrm{H}]^{+} 386.2331$, found: $386.2344 ;[\alpha]^{23}{ }_{\mathrm{D}}=-79^{\circ}(c 0.12, \mathrm{MeOH})$.

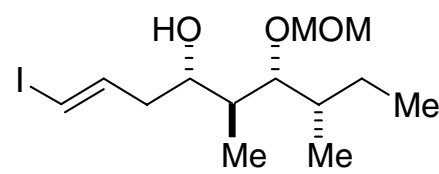

$(1 E, 4 S, 5 R, 6 R, 7 S)-5,7-D i m e t h y l-1-i o d o-4-$ methoxymethoxy-oct-1-en-4-ol (19): A stirred solution of

$4(15 \mathrm{mg}, 0.029 \mathrm{mmol})$ in THF $(1.5 \mathrm{~mL}, 0.02 \mathrm{M})$ in a plastic vial was treated with pyridine $(0.6 \mathrm{~mL}, 7.4 \mathrm{mmol}, 255$ equiv.) followed by $70 \%$ HF/pyridine (0.6 M, 1:1 vol. equiv. vs. pyridine). The solution was stirred $68 \mathrm{~h}$ and quenched with water. The aqueous layer was extracted into EtOAc, dried over $\mathrm{MgSO}_{4}$, filtered, and concentrated under reduced pressure. Purification by flash chromatography (silica, 10\% EtOAc/hexanes) gave secondary alcohol 19 (10.0 mg, $96 \%)$ as a yellow oil. ${ }^{1} \mathrm{H}$ NMR $\left(270 \mathrm{MHz}, \mathrm{CDCl}_{3}\right) \delta 6.64(\mathrm{dt}, J=7.8,14.2 \mathrm{~Hz}, 2 \mathrm{H})$, $6.08(\mathrm{~d}, J=14.2 \mathrm{~Hz}, 1 \mathrm{H}), 4.64$ (s, 2H), 3.69 (m, 1H), 3.39 (s, 3H), 3.33 (dd, $J=2.4,7.8$ $\mathrm{Hz}, 1 \mathrm{H}), 3.28(\mathrm{~d}, J=2.9 \mathrm{~Hz}, 1 \mathrm{H}), 2.35(\mathrm{~m}, 1 \mathrm{H}), 2.15(\mathrm{~m}, 1 \mathrm{H}), 1.78(\mathrm{~m}, 1 \mathrm{H}), 1.52(\mathrm{~m}$, 1H), $1.40(\mathrm{~m}, 1 \mathrm{H}), 1.24(\mathrm{~m}, 1 \mathrm{H}), 0.90$ (t, $J=7.8 \mathrm{~Hz}, 3 \mathrm{H}), 0.88$ (d, $J=6.8 \mathrm{~Hz}, 3 \mathrm{H}), 0.83$ $(\mathrm{d}, J=6.8 \mathrm{~Hz}, 3 \mathrm{H}) ;{ }^{13} \mathrm{C}$ NMR $\left(67.9 \mathrm{MHz}, \mathrm{CDCl}_{3}\right) \delta 143.5,98.5,87.0,76.3,72.6,56.3$, 41.0, 40.3, 38.3, 27.2, 14.5, 13.5, 12.2; IR (neat) $v_{\max } 3446,2963,2932,1457,1380$, 1139, 1089, 1037; HRMS (CI, $\left.\mathrm{NH}_{3}\right), m / z$ calculated for $\mathrm{C}_{13} \mathrm{H}_{26} \mathrm{IO}_{3}[\mathrm{M}+\mathrm{H}]^{+} 357.0927$, found: $357.0926 ;[\alpha]^{23}=-15^{\circ}\left(c 0.28, \mathrm{CHCl}_{3}\right)$.

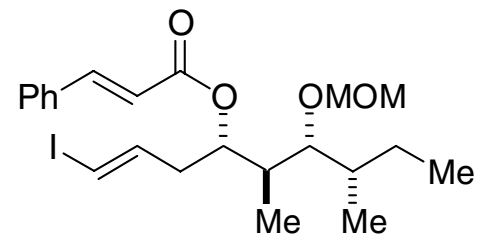

$(1 E, 4 S, 5 R, 6 R, 7 S)$-1-Iodo-5,7-dimethyl-4-[(2E)-3phenylacryloxy]-6-methoxymethoxy-oct-1-ene (20): Alcohol 19 (8.6 mg, $0.0241 \mathrm{mmol}$ ) was dissolved in $\mathrm{CH}_{2} \mathrm{Cl}_{2}(0.5 \mathrm{~mL}, 0.05 \mathrm{M})$ and treated with triethylamine (0.013 mL, $0.0966 \mathrm{mmol}, 4.0$ equiv.), followed by DMAP ( $0.3 \mathrm{mg}, 0.00241 \mathrm{mmol}, 0.1$ equiv.). The solution was cooled to $0{ }^{\circ} \mathrm{C}$ and $(E)$-cinnamoyl chloride ( $8.0 \mathrm{mg}, 0.0483$ mmol, 2.0 equiv.) was added. The solution was protected from light and stirred $48 \mathrm{~h}$ and additional amounts of triethylamine $(0.013 \mathrm{~mL}, 0.0966 \mathrm{mmol}, 4.0$ equiv.) and (E)cinnamoyl chloride ( $8.0 \mathrm{mg}, 0.0483 \mathrm{mmol}, 2.0$ equiv.) were added. The reaction was stirred an additional $48 \mathrm{~h}$ and quenched with saturated $\mathrm{NaHCO}_{3}$. The aqueous phase 
was extracted into $\mathrm{CH}_{2} \mathrm{Cl}_{2}(3 \times)$. The combined organic extracts were washed with brine, dried over $\mathrm{MgSO}_{4}$, filtered, and concentrated in vacuo. Purification by flash chromatography (silica, $10 \% \mathrm{EtOAc} / \mathrm{CH}_{2} \mathrm{Cl}_{2}$ ) yielded the cinnamate ester $20(10.0 \mathrm{mg}$, $85 \%)$ as a pale yellow oil. ${ }^{1} \mathrm{H}$ NMR $\left(270 \mathrm{MHz}, \mathrm{CDCl}_{3}\right) \delta 7.66(\mathrm{~d}, J=16.1 \mathrm{~Hz}, 1 \mathrm{H})$, $7.50(\mathrm{~m}, 2 \mathrm{H}), 7.40(\mathrm{~m}, 3 \mathrm{H}), 6.50(\mathrm{~m}, 1 \mathrm{H}), 6.41(\mathrm{~d}, J=16.1 \mathrm{~Hz}, 1 \mathrm{H}), 6.09(\mathrm{~d}, J=14.7$ $\mathrm{Hz}, 1 \mathrm{H}), 5.32(\mathrm{~m}, 1 \mathrm{H}), 4.63(\mathrm{~s}, 2 \mathrm{H}), 3.43(\mathrm{~s}, 3 \mathrm{H}), 3.30(\mathrm{dd}, J=2.9,7.8), 6.45(\mathrm{~m}, 1 \mathrm{H})$, $2.33(\mathrm{~m}, 1 \mathrm{H}), 2.15(\mathrm{~m}, 1 \mathrm{H}), 1.53(\mathrm{~m}, 1 \mathrm{H}), 1.42(\mathrm{~m}, 1 \mathrm{H}), 1.24(\mathrm{~m}, 1 \mathrm{H}), 0.91(\mathrm{t}, J=7.3$, $3 \mathrm{H}), 0.86(\mathrm{~d}, J=6.4 \mathrm{~Hz}, 3 \mathrm{H}), 0.86(\mathrm{~d}, J=6.4 \mathrm{~Hz}, 3 \mathrm{H}) ;{ }^{13} \mathrm{C} \mathrm{NMR}\left(67.9 \mathrm{MHz}, \mathrm{CDCl}_{3}\right) \delta$ 166.3, 144.8, 142.3, 134.4, 133.3, 128.9, 128.1, 118.3, 98.4, 98.4, 83.9, 76.8, 73.2, 56.3, 38.9, 37.0, 36.1, 27.3, 13.4, 12.1, 11.5; IR (neat) $v_{\max } 2964,2933,1711,1636,1167$, 1037, 767; HRMS (CI, $\mathrm{NH}_{3}$ ), $m / z$ calculated for $\mathrm{C}_{22} \mathrm{H}_{32} \mathrm{IO}_{4}[\mathrm{M}+\mathrm{H}]^{+} 487.1345$, found: $487.1393 ;[\alpha]^{23}{ }_{\mathrm{D}}=-54^{\circ}\left(c 0.5, \mathrm{CHCl}_{3}\right)$.

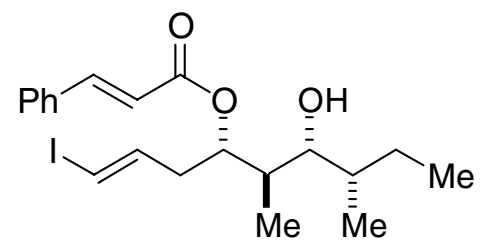

$(1 E, 4 S, 5 R, 6 R, 7 S)-5,7-d i m e t h y l-1-i o d o-4-[(2 E)-3-$ phenylacryloxy]-oct-1-en-6-ol (21): A solution of 20 (8.9 mg, $0.0189 \mathrm{mmol})$ was prepared in $\mathrm{CH}_{2} \mathrm{Cl}_{2}(1.00 \mathrm{~mL}$, $0.019 \mathrm{M})$, cooled to $-78{ }^{\circ} \mathrm{C}$, and treated with $\mathrm{BCl}_{3}(0.04$ $\mathrm{mL}$ of $1 \mathrm{M}$ solution in hexanes, 2.0 equiv.). The solution was protected from light, stirred for $4 \mathrm{~h}$ and quenched with the addition of $\mathrm{MeOH}(0.50 \mathrm{~mL})$ followed by saturated aqueous $\mathrm{NaHCO}_{3}$. The aqueous phase was extracted into $\mathrm{CH}_{2} \mathrm{Cl}_{2}(3 \times)$. The combined organic extracts were washed with brine, dried over $\mathrm{MgSO}_{4}$, filtered, and concentrated in vacuo. Purification by flash chromatography (silica, 10\% EtOAc/ $\left.\mathrm{CH}_{2} \mathrm{Cl}_{2}\right)$ yielded the secondary alcohol $20(6.1 \mathrm{mg}, 69 \%)$ as a pale yellow oil. ${ }^{1} \mathrm{H}$ NMR (400 MHz, $\left.\mathrm{CDCl}_{3}\right) \delta 7.61(\mathrm{~d}, J=16.0 \mathrm{~Hz}, 1 \mathrm{H}), 7.47(\mathrm{~m}, 2 \mathrm{H}), 7.32(\mathrm{~m}, 3 \mathrm{H})$, $6.47(\mathrm{dt}, J=7.6,14.6 \mathrm{~Hz}, 1 \mathrm{H}), 6.37(\mathrm{~d}, J=16.0 \mathrm{~Hz}, 1 \mathrm{H}), 6.06(\mathrm{~d}, J=14.6 \mathrm{~Hz}, 1 \mathrm{H})$, $5.32(\mathrm{dt}, J=4.0,8.8 \mathrm{~Hz}, 1 \mathrm{H}), 3.34(\mathrm{~m}, 1 \mathrm{H}), 2.34(\mathrm{~m}, 2 \mathrm{H}), 2.00(\mathrm{~m}, 1 \mathrm{H}), 1.41(\mathrm{~m}, 1 \mathrm{H})$, $1.37(\mathrm{~m}, 1 \mathrm{H}), 1.27(\mathrm{~m}, 2 \mathrm{H}), 0.86(\mathrm{t}, J=7.2 \mathrm{~Hz}, 3 \mathrm{H}), 0.82(\mathrm{~d}, J=6.8 \mathrm{~Hz}, 3 \mathrm{H}), 0.76(\mathrm{~d}, J$ $=6.8 \mathrm{~Hz}, 3 \mathrm{H}) ;{ }^{13} \mathrm{C} \mathrm{NMR}\left(67.9 \mathrm{MHz}, \mathrm{CDCl}_{3}\right) \delta 166.4,145.0,142.3,134.3,128.9,128.1$, $118.1,77.1,75.6,73.9,39.4,36.6,35.9,27.0,12.0,12.0,11.6,11.3$; IR (neat) $v_{\max } 3446$, 
2963, 2933, 1701, 1636, 1203, 1172, 989; HRMS (CI, $\mathrm{NH}_{3}$ ), m/z calculated for $\mathrm{C}_{13} \mathrm{H}_{26} \mathrm{IO}_{3}[\mathrm{M}+\mathrm{H}]^{+} 357.0927$, found: 443.1119; $[\alpha]^{23}{ }_{\mathrm{D}}=-77^{\circ}\left(c 0.30, \mathrm{CHCl}_{3}\right)$.

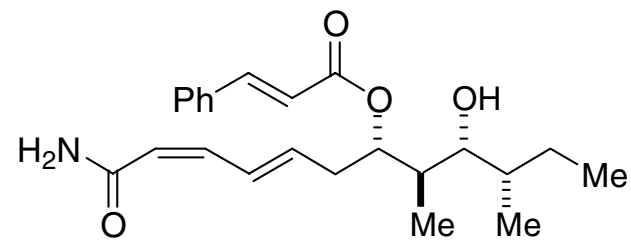

Basiliskamide B (1b): To 21 (5.1 mg, 0.0115 mmol) was added a solution of stannane $3(0.20$ $\mathrm{mL}$ of $0.08 \mathrm{M}$ solution in DMF, 1.4 equiv.) and dichlor(bis)acetonitrile palladium(II) $(0.15 \mathrm{mg}$ in $0.04 \mathrm{~mL}$ DMF, $0.00058 \mathrm{mmol}, 0.05$ equiv.). After $18 \mathrm{~h}$, the reaction was quenched with $\mathrm{H}_{2} \mathrm{O}$. The mixture was diluted with EtOAc and washed with $\mathrm{H}_{2} \mathrm{O}(3 \times)$. The combined aqueous washings were reextracted with EtOAc. The combined organic extracts were then washed with brine, dried over $\mathrm{MgSO}_{4}$, filtered, and concentrated in vacuo. Purification by flash chromatography (silica, $75 \% \mathrm{EtOAc} / \mathrm{CH}_{2} \mathrm{Cl}_{2}$ ) afforded synthetic basiliskamide B 1b (3.4 mg, 76\%) as a colorless solid. ${ }^{1} \mathrm{H}$ NMR (400 MHz, DMSO- $\left.d_{6}\right) \delta 7.71(\mathrm{~m}, 2 \mathrm{H}), 7.62(\mathrm{~d}, J=16.0 \mathrm{~Hz}, 1 \mathrm{H}), 7.53(\mathrm{dd}, J=11.0,15.0 \mathrm{~Hz}, 1 \mathrm{H})$, $7.41(\mathrm{~m}, 3 \mathrm{H}), 7.39$ (s, 1H), 6.91 (s, 1H), $6.60(\mathrm{~d}, J=16.0 \mathrm{~Hz}, 1 \mathrm{H}), 6.34$ (dd, $J=11.0$, $11.0 \mathrm{~Hz}, 1 \mathrm{H}), 5.88(\mathrm{dt}, J=7.2,15.0 \mathrm{~Hz}, 1 \mathrm{H}), 5.58(\mathrm{~d}, J=11.0 \mathrm{~Hz}, 1 \mathrm{H}), 5.41(\mathrm{dt}, J=$ 3.1, $10.4 \mathrm{~Hz}, 1 \mathrm{H}), 4.51(\mathrm{br} \mathrm{s}, 1 \mathrm{H}), 3.27(\mathrm{~m}, 1 \mathrm{H}), 2.53(\mathrm{~m}, 1 \mathrm{H}), 2.36(\mathrm{~m}, 1 \mathrm{H}), 1.92(\mathrm{~m}$, $1 \mathrm{H}), 1.40(\mathrm{~m}, 1 \mathrm{H}), 1.38(\mathrm{~m}, 1 \mathrm{H}), 1.21(\mathrm{~m}, 1 \mathrm{H}), 0.86(\mathrm{t}, J=7.2 \mathrm{~Hz}, 3 \mathrm{H}), 0.84(\mathrm{~d}, J=6.8$ $\mathrm{Hz}, 3 \mathrm{H}), 0.75(\mathrm{~d}, J=6.4 \mathrm{~Hz}, 3 \mathrm{H}) ;{ }^{13} \mathrm{C}$ NMR $\left(67.9 \mathrm{MHz}, \mathrm{DMSO}-d_{6}\right) \delta 168.6,166.8$, 145.3, 141.3, 139.3, 135.2, 131.5, 130.2, 130.0, 129.5, 121.0, 119.6, 75.1, 74.0, 37.3, 32.8, 27.6, 13.1, 12.9, 11.6; IR (neat) $v_{\max } 3348,3198,2956,2924,2854,1700,1668$, 1636, 1457, 1182, 992, 766; $\mathrm{m} / \mathrm{z}$ calculated for $\mathrm{C}_{23} \mathrm{H}_{32} \mathrm{NO}_{4}[\mathrm{M}+\mathrm{H}]^{+} 386.2331$, found: $386.2314 ;[\alpha]^{23}{ }_{\mathrm{D}}=-14^{\circ}(c 0.20, \mathrm{MeOH})$. 


\section{Table I. Comparison of ${ }^{1} \mathrm{H}$ and ${ }^{13} \mathrm{C}$ NMR Data between Natural ${ }^{6}$ and Synthetic Basiliskamide A}

\begin{tabular}{|c|c|c|c|c|c|}
\hline $\begin{array}{c}\text { Natural } \\
\text { Basilskamide A }\end{array}$ & $\begin{array}{c}\text { Synthetic } \\
\text { Basiliskamide A }\end{array}$ & & $\begin{array}{c}\text { Natural } \\
\text { Basilskamide A }\end{array}$ & $\begin{array}{c}\text { Synthetic } \\
\text { Basiliskamide A }\end{array}$ & \\
\hline $\begin{array}{c}{ }^{\mathrm{T}} \mathrm{H} \text { NMR }\left(\mathrm{DMSO}-d_{6}\right) \\
500 \mathrm{MHz}, \delta(\mathrm{ppm})\end{array}$ & $\begin{array}{c}{ }^{\mathrm{T}} \mathrm{H} \text { NMR }\left(\mathrm{DMSO}-d_{6}\right) \\
400 \mathrm{MHz}, \delta(\mathrm{ppm})\end{array}$ & $\begin{array}{c}\Delta \delta \\
(\mathrm{ppm})\end{array}$ & $\begin{array}{c}{ }^{13} \mathrm{C} \text { NMR }\left(\mathrm{DMSO}-d_{6}\right) \\
100 \mathrm{MHz}, \delta(\mathrm{ppm}) \\
\end{array}$ & $\begin{array}{c}{ }^{13} \mathrm{C} \text { NMR }\left(\mathrm{DMSO}-d_{6}\right) \\
75 \mathrm{MHz}, \delta(\mathrm{ppm}) \\
\end{array}$ & $\begin{array}{c}\Delta \delta \\
(\mathrm{ppm})\end{array}$ \\
\hline $0.84(\mathrm{~d}, J=7.0 \mathrm{~Hz}, 3 \mathrm{H})$ & $0.83(\mathrm{~d}, J=6.8 \mathrm{~Hz}, 3 \mathrm{H})$ & -0.01 & 10.1 & 10.5 & 50.4 \\
\hline $0.87(\mathrm{t}, J=7.5 \mathrm{~Hz}, 3 \mathrm{H})$ & $0.87(\mathrm{t}, J=7.6 \mathrm{~Hz}, 3 \mathrm{H})$ & 0.0 & 11.6 & 12.2 & 0.6 \\
\hline $0.90(\mathrm{~d}, J=7.0 \mathrm{~Hz}, 3 \mathrm{H})$ & $0.90(\mathrm{~d}, J=7.2 \mathrm{~Hz}, 3 \mathrm{H})$ & 0.0 & 12.8 & 13.3 & 0.5 \\
\hline $1.11(\mathrm{~m}, 1 \mathrm{H})$ & $1.11(\mathrm{~m}, 1 \mathrm{H})$ & 0.0 & 26.4 & 27.0 & 0.6 \\
\hline $1.25(\mathrm{~m}, 1 \mathrm{H})$ & $1.25(\mathrm{~m}, 1 \mathrm{H})$ & 0.0 & 34.7 & 35.1 & 0.4 \\
\hline $1.67(\mathrm{~m}, 1 \mathrm{H})$ & $1.68(\mathrm{~m}, 1 \mathrm{H})$ & 0.01 & 35.5 & 36.0 & 0.5 \\
\hline $1.99(\mathrm{~m}, 1 \mathrm{H})$ & $1.99(\mathrm{~m}, 1 \mathrm{H})$ & 0.0 & 40.7 & 41.3 & \begin{tabular}{|l|l|}
3 & 0.4 \\
\end{tabular} \\
\hline $2.06(\mathrm{~m}, 1 \mathrm{H})$ & $2.01(\mathrm{~m}, 1 \mathrm{H})$ & -0.05 & 69.6 & 70.2 & 0.4 \\
\hline $2.28(\mathrm{~m}, 1 \mathrm{H})$ & $2.28(\mathrm{~m}, 1 \mathrm{H})$ & 0.0 & 76.3 & 76.8 & 0.5 \\
\hline $3.49(\mathrm{~m}, 1 \mathrm{H})$ & $3.47(\mathrm{~m}, 1 \mathrm{H})$ & -0.02 & 118.0 & 118.6 & 0.4 \\
\hline $4.57(\mathrm{~d}, J=5.0 \mathrm{~Hz}, 1 \mathrm{H})$ & $4.61(\mathrm{~d}, J=5.2 \mathrm{~Hz}, 1 \mathrm{H})$ & 0.04 & 119.3 & 120.0 & 0.7 \\
\hline $4.92(\mathrm{dd}, J=2.0,9.5 \mathrm{~Hz}, 1 \mathrm{H})$ & $4.92(\mathrm{~m}, 1 \mathrm{H})$ & 0.0 & 128.2 & 129.0 & 0.8 \\
\hline $5.55(\mathrm{~d}, J=11.0 \mathrm{~Hz}, 1 \mathrm{H})$ & $5.55(\mathrm{~d}, J=11.6 \mathrm{~Hz}, 1 \mathrm{H})$ & 0.0 & 128.4 & 129.1 & 0.7 \\
\hline $5.91(\mathrm{dt}, J=7.0,15.0 \mathrm{~Hz}, 1 \mathrm{H})$ & $5.90(\mathrm{dt}, J=7.3,14.6 \mathrm{~Hz}, 1 \mathrm{H})$ & $\mid-0.01$ & 128.9 & 129.6 & 0.7 \\
\hline $6.31(\mathrm{dd}, J=11.0,11.0 \mathrm{~Hz}, 1 \mathrm{H})$ & $6.30(\mathrm{dt}, J=11.3,11.6 \mathrm{~Hz}, 1 \mathrm{H})$ & -0.01 & 130.4 & 131.1 & 0.7 \\
\hline $6.61(\mathrm{~d}, J=16.0 \mathrm{~Hz}, 1 \mathrm{H})$ & $6.63(\mathrm{~d}, J=15.9 \mathrm{~Hz}, 1 \mathrm{H})$ & 0.02 & 134.0 & 134.7 & 70.7 \\
\hline $6.83(\mathrm{~s}, 1 \mathrm{H})$ & $6.88(\mathrm{~s}, 1 \mathrm{H})$ & 0.05 & 140.5 & 141.3 & \begin{tabular}{|l|l|}
3 & 0.8 \\
\end{tabular} \\
\hline $7.31(\mathrm{~s}, 1 \mathrm{H})$ & $7.36(\mathrm{~s}, 1 \mathrm{H})$ & 0.05 & 114.6 & 145.4 & 0.8 \\
\hline $7.40(\mathrm{dd}, J=11.0,15.0 \mathrm{~Hz}, 1 \mathrm{H})$ & $7.40($ par obsc $\mathrm{m}, 1 \mathrm{H})$ & 0.0 & 166.0 & 166.8 & \begin{tabular}{|l|l|}
30.8 \\
\end{tabular} \\
\hline $7.40(\mathrm{~m}, 1 \mathrm{H})$ & $7.40(\mathrm{~m}, 3 \mathrm{H})$ & 0.0 & 167.4 & 168.3 & 0.9 \\
\hline \multicolumn{6}{|l|}{$7.41(\mathrm{~m}, 2 \mathrm{H})$} \\
\hline $7.65(\mathrm{~d}, J=16.0 \mathrm{~Hz}, 1 \mathrm{H})$ & $7.66(\mathrm{~d}, J=15.9 \mathrm{~Hz}, 1 \mathrm{H})$ & 0.01 & & & \\
\hline $7.71(\mathrm{~m}, 2 \mathrm{H})$ & $7.72(\mathrm{~m}, 2 \mathrm{H})$ & 0.01 & & & \\
\hline
\end{tabular}

${ }^{6}$ NMR data for natural basiliskamides A and B obtained from isolation article: Barsby, T.; Kelly, M. T.; Andersen, R. J. J. Nat. Prod. 2002, 65, 1447-1451. 
Table II. Comparison of ${ }^{1} \mathrm{H}$ and ${ }^{13} \mathrm{C}$ NMR Data between Natural and Synthetic Basiliskamide B

\begin{tabular}{|c|c|c|c|c|c|}
\hline $\begin{array}{c}\text { Natural } \\
\text { Basilskamide B }\end{array}$ & $\begin{array}{c}\text { Synthetic } \\
\text { Basiliskamide B }\end{array}$ & & $\begin{array}{c}\text { Natural } \\
\text { Basilskamide B }\end{array}$ & $\begin{array}{c}\text { Synthetic } \\
\text { Basiliskamide B }\end{array}$ & \\
\hline $\begin{array}{c}{ }^{1} \mathrm{H} \text { NMR }\left(\mathrm{DMSO}-d_{6}\right) \\
500 \mathrm{MHz}, \delta(\mathrm{ppm})\end{array}$ & $\begin{array}{c}{ }^{1} \mathrm{H} \text { NMR }\left(\mathrm{DMSO}-d_{6}\right) \\
400 \mathrm{MHz}, \delta(\mathrm{ppm})\end{array}$ & $\begin{array}{c}\Delta \delta \\
(\mathrm{ppm})\end{array}$ & $\begin{array}{c}\left.{ }^{13} \mathrm{C} \text { NMR (DMSO- } d_{6}\right) \\
100 \mathrm{MHz}, \delta(\mathrm{ppm})\end{array}$ & $\begin{array}{c}\left.{ }^{13} \mathrm{C} \text { NMR (DMSO- } d_{6}\right) \\
75 \mathrm{MHz}, \delta(\mathrm{ppm}) \\
\end{array}$ & $\begin{array}{c}\Delta \delta \\
(\mathrm{ppm})\end{array}$ \\
\hline $0.74(\mathrm{~d}, J=7.0 \mathrm{~Hz}, 3 \mathrm{H})$ & $0.75(\mathrm{~d}, J=6.4 \mathrm{~Hz}, 3 \mathrm{H})$ & 0.01 & 10.7 & 11.6 & 0.9 \\
\hline $0.83(\mathrm{~d}, J=7.0 \mathrm{~Hz}, 3 \mathrm{H})$ & $0.84(\mathrm{~d}, J=6.8 \mathrm{~Hz}, 3 \mathrm{H})$ & 0.01 & 11.8 & 12.9 & 0.9 \\
\hline $0.87(\mathrm{~d}, J=7.0 \mathrm{~Hz}, 3 \mathrm{H})$ & $0.86(\mathrm{t}, J=7.2 \mathrm{~Hz}, 3 \mathrm{H})$ & -0.01 & 12.1 & 13.1 & 1.0 \\
\hline $1.21(\mathrm{~m}, 1 \mathrm{H})$ & $1.21(\mathrm{~m}, 1 \mathrm{H})$ & 0.0 & 26.5 & 27.6 & 0.9 \\
\hline $1.38(\mathrm{~m}, 1 \mathrm{H})$ & $1.38(\mathrm{~m}, 1 \mathrm{H})$ & 0.0 & 31.8 & 32.8 & 1.0 \\
\hline $1.40(\mathrm{~m}, 1 \mathrm{H})$ & $1.40(\mathrm{~m}, 1 \mathrm{H})$ & 0.0 & 36.3 & 37.3 & 1.0 \\
\hline $1.92(\mathrm{~m}, 1 \mathrm{H})$ & $1.92(\mathrm{~m}, 1 \mathrm{H})$ & 0.0 & 73.0 & 74.0 & 1.0 \\
\hline $2.36(\mathrm{~m}, 1 \mathrm{H})$ & $2.36(\mathrm{~m}, 1 \mathrm{H})$ & 0.0 & 74.0 & 75.1 & 1.1 \\
\hline $2.53(\mathrm{~m}, 1 \mathrm{H})$ & $2.53(\mathrm{~m}, 1 \mathrm{H})$ & 0.0 & 118.5 & 119.6 & 1.1 \\
\hline $3.26(\mathrm{~m}, 1 \mathrm{H})$ & $3.27(\mathrm{~m}, 1 \mathrm{H})$ & 0.01 & 119.9 & 121.0 & 1.1 \\
\hline $4.48(\mathrm{~m}, 1 \mathrm{H})$ & $4.51(\mathrm{br} \mathrm{s}, 1 \mathrm{H})$ & 0.03 & 128.2 & 129.5 & 1.3 \\
\hline $5.40(\mathrm{dt}, J=3.0,10.5 \mathrm{~Hz}, 1 \mathrm{H})$ & $5.41(\mathrm{dt}, J=3.2,10.4,1 \mathrm{H})$ & 0.01 & 128.8 & 130.0 & 1.2 \\
\hline $5.57(\mathrm{~d}, J=11.0 \mathrm{~Hz}, 1 \mathrm{H})$ & $5.58(\mathrm{~d}, J=11.0 \mathrm{~Hz}, 1 \mathrm{H})$ & 0.01 & 129.0 & 130.2 & 1.2 \\
\hline $5.87(\mathrm{dt}, J=7.0,15.0 \mathrm{~Hz}, 1 \mathrm{H})$ & $5.88(\mathrm{dt}, J=7.2,15.0 \mathrm{~Hz}, 1 \mathrm{H})$ & 0.01 & 130.2 & 131.5 & 1.3 \\
\hline $6.33(\mathrm{dd}, J=11.0,11.0 \mathrm{~Hz}, 1 \mathrm{H})$ & $6.34(\mathrm{dd}, J=11.0,11.0 \mathrm{~Hz}, 1 \mathrm{H})$ & 0.01 & 134.0 & 135.2 & 1.2 \\
\hline $6.59(\mathrm{~d}, J=16.0 \mathrm{~Hz}, 1 \mathrm{H})$ & $6.60(\mathrm{~d}, J=16.0 \mathrm{~Hz}, 1 \mathrm{H})$ & 0.01 & 138.0 & 139.3 & 1.3 \\
\hline $6.86(\mathrm{~s}, 1 \mathrm{H})$ & $6.91(\mathrm{~s}, 1 \mathrm{H})$ & 0.05 & 140.0 & 141.3 & 1.3 \\
\hline $7.34(\mathrm{~s}, 1 \mathrm{H})$ & $7.38(\mathrm{~s}, 1 \mathrm{H})$ & 0.04 & 144.1 & 145.3 & 1.2 \\
\hline $7.40(\mathrm{~m}, 3 \mathrm{H})$ & $7.41(\mathrm{~m}, 3 \mathrm{H})$ & 0.01 & 165.5 & 166.8 & 1.3 \\
\hline $7.51(\mathrm{dd}, J=11.0,15.0 \mathrm{~Hz}, 1 \mathrm{H})$ & $7.53(\mathrm{dd}, J=11.0,15.0 \mathrm{~Hz}, 1 \mathrm{H})$ & 0.02 & 167.4 & 168.6 & 1.2 \\
\hline $7.60(\mathrm{~d}, J=16.0 \mathrm{~Hz}, 1 \mathrm{H})$ & $7.62(\mathrm{~d}, J=16.0 \mathrm{~Hz}, 1 \mathrm{H})$ & 0.02 & & & \\
\hline $7.70(\mathrm{~m}, 2 \mathrm{H})$ & $7.71(\mathrm{~m}, 2 \mathrm{H})$ & 0.01 & & & \\
\hline
\end{tabular}


${ }^{1}$ H NMR (400 MHz, DMSO- $d_{6}$ ) of synthetic Basiliskamide A 


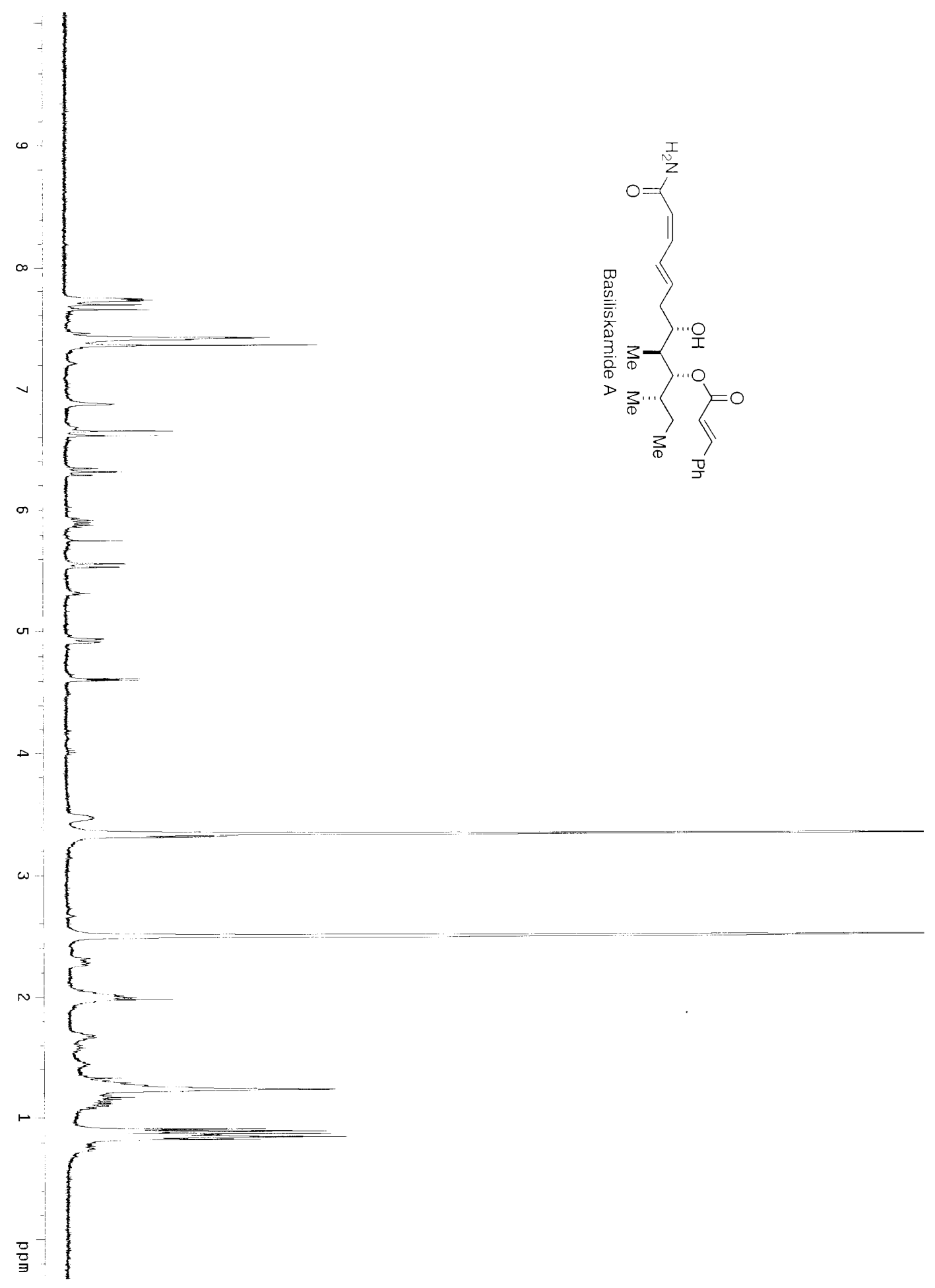


${ }^{1}$ H NMR (400 MHz, DMSO- $\left.d_{6}\right)$ of natural Basiliskamide $A^{7}$ 04/18/04 SUN 16:35 FAX 604 822 6091 EOS/OCEANOGRAPHY

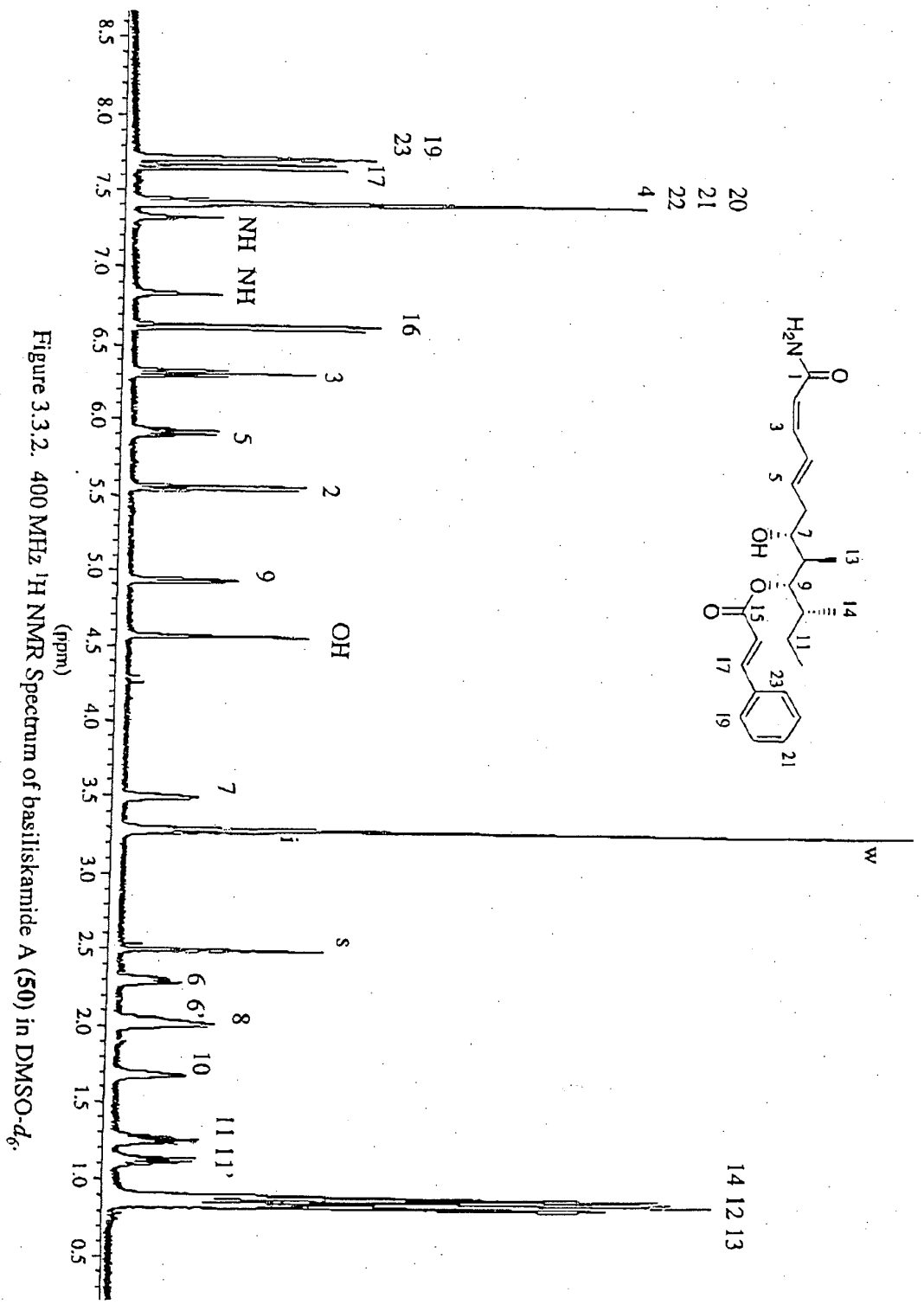

I6

\footnotetext{
${ }^{71} \mathrm{H}$ and ${ }^{13} \mathrm{C}$ NMR spectra of natural basiliskamides kindly provided by Professor Raymond J. Andersen.
} 


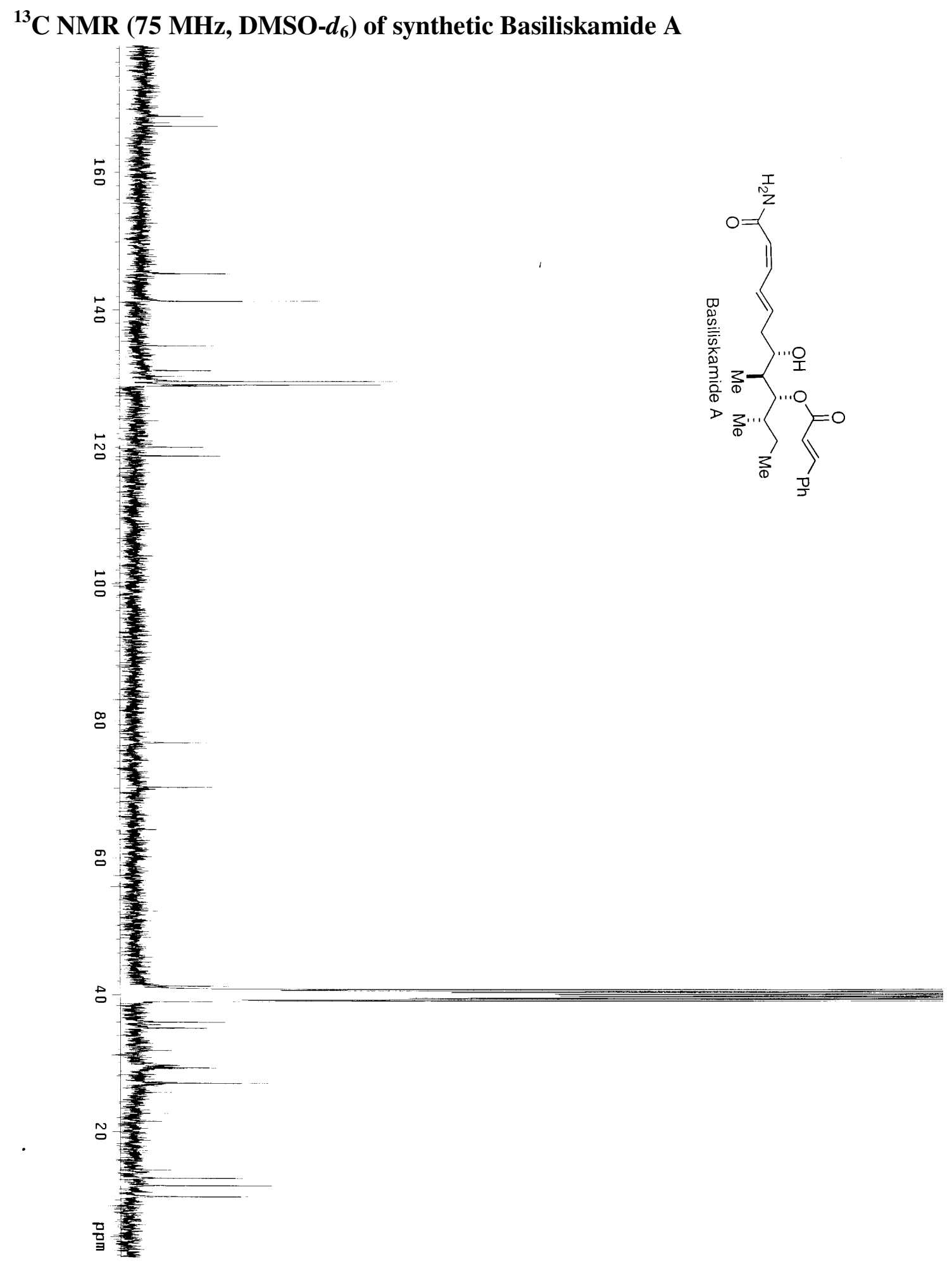


${ }^{13}$ C NMR (100 MHz, DMSO- $d_{6}$ ) of natural Basiliskamide A $04 / 18 / 04$ SIN $18: 35$ FAX $604 \quad 822 \quad 8091$

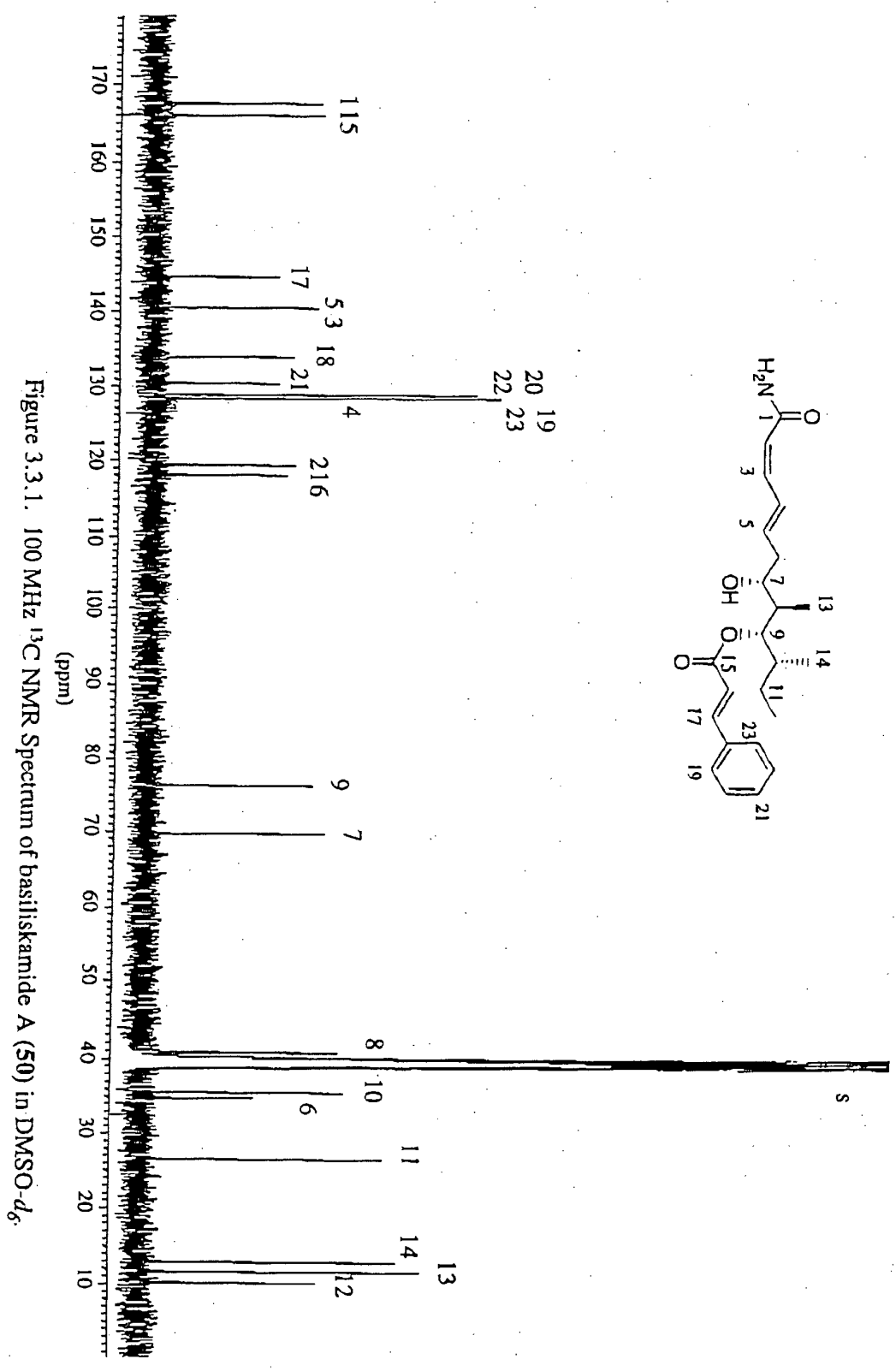




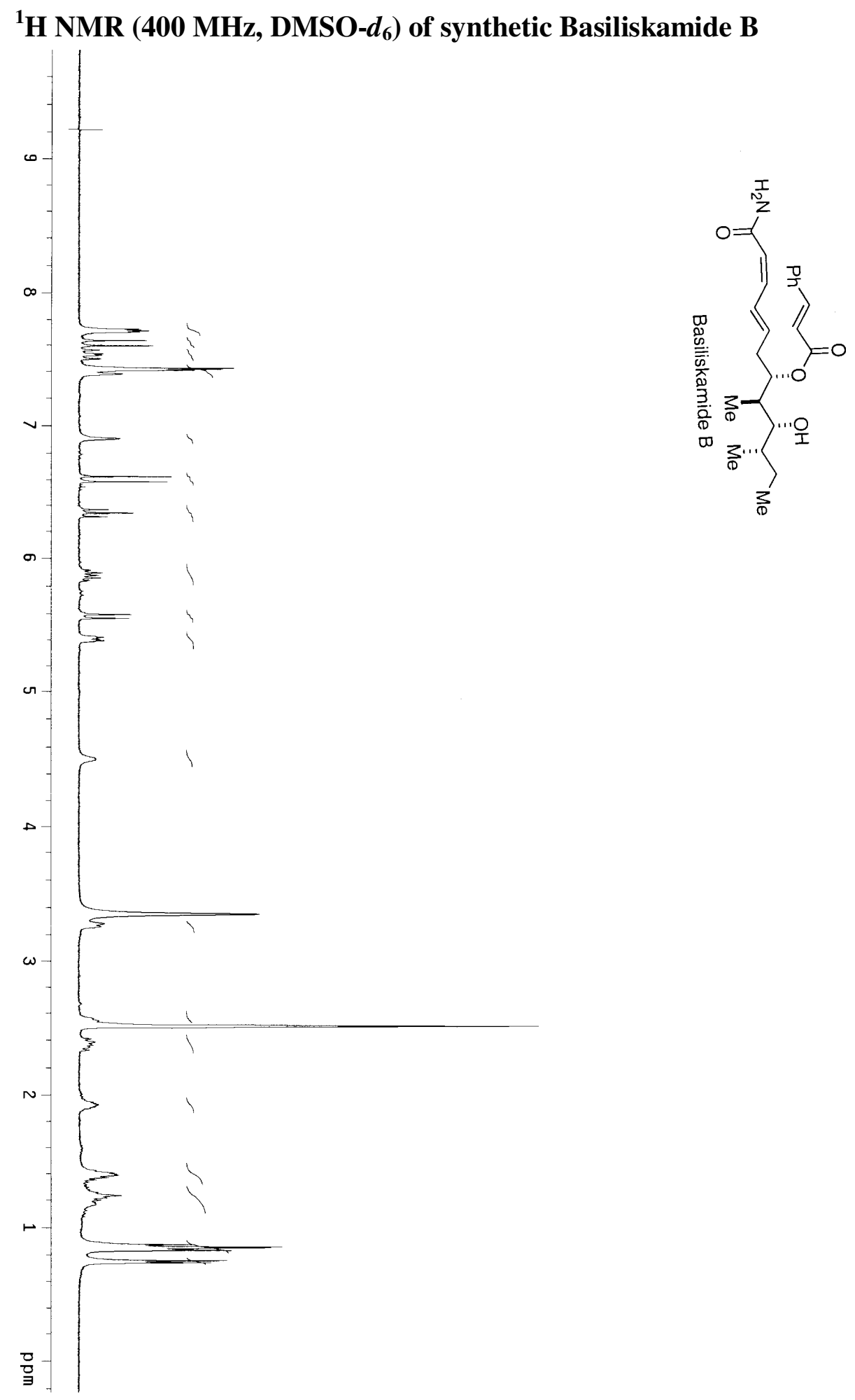


${ }^{1} \mathrm{H}$ NMR (400 MHz, DMSO- $d_{6}$ ) of natural Basiliskamide B 04/18/04 SUN 16:35 FAX 604 8226091

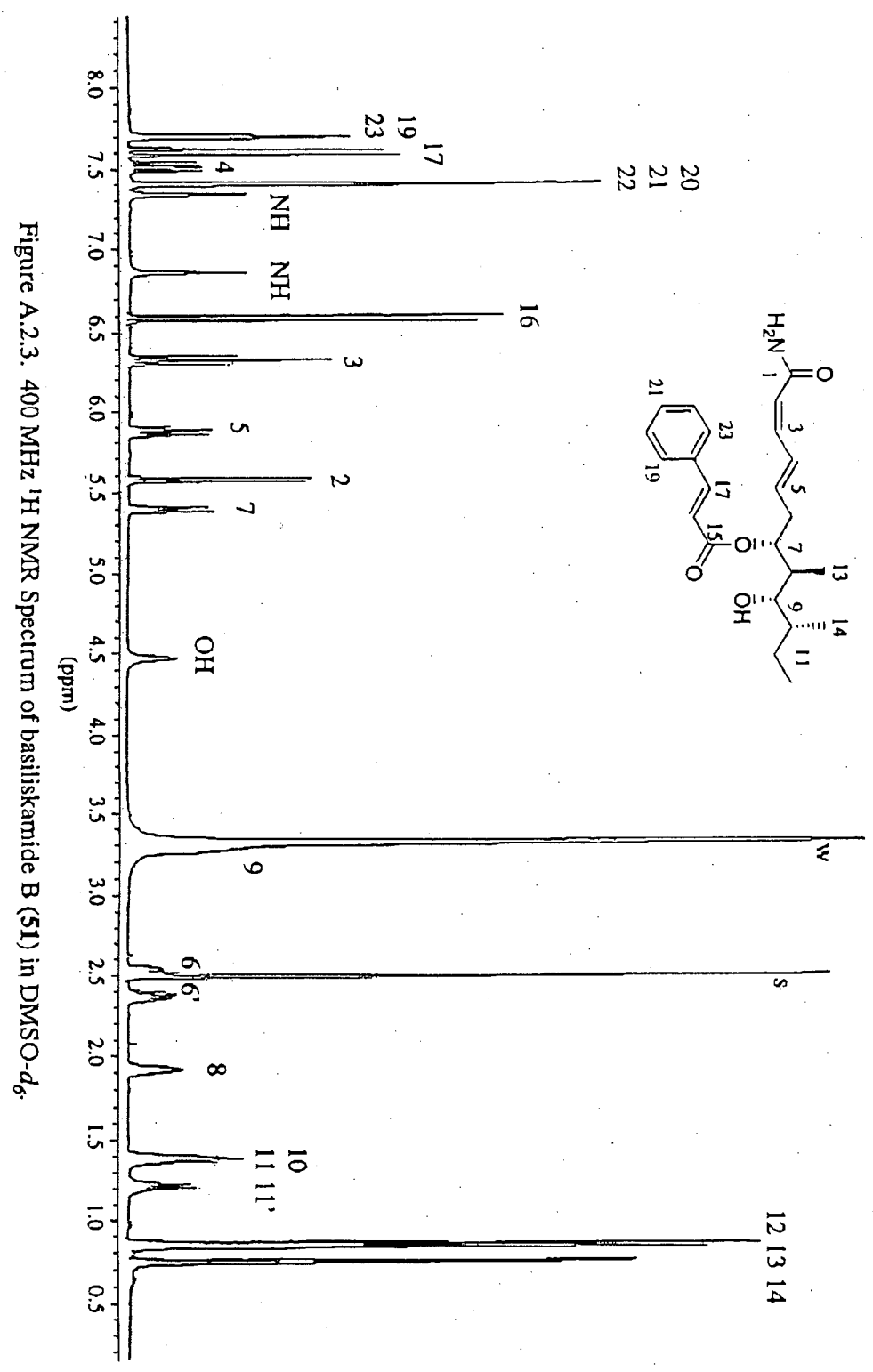




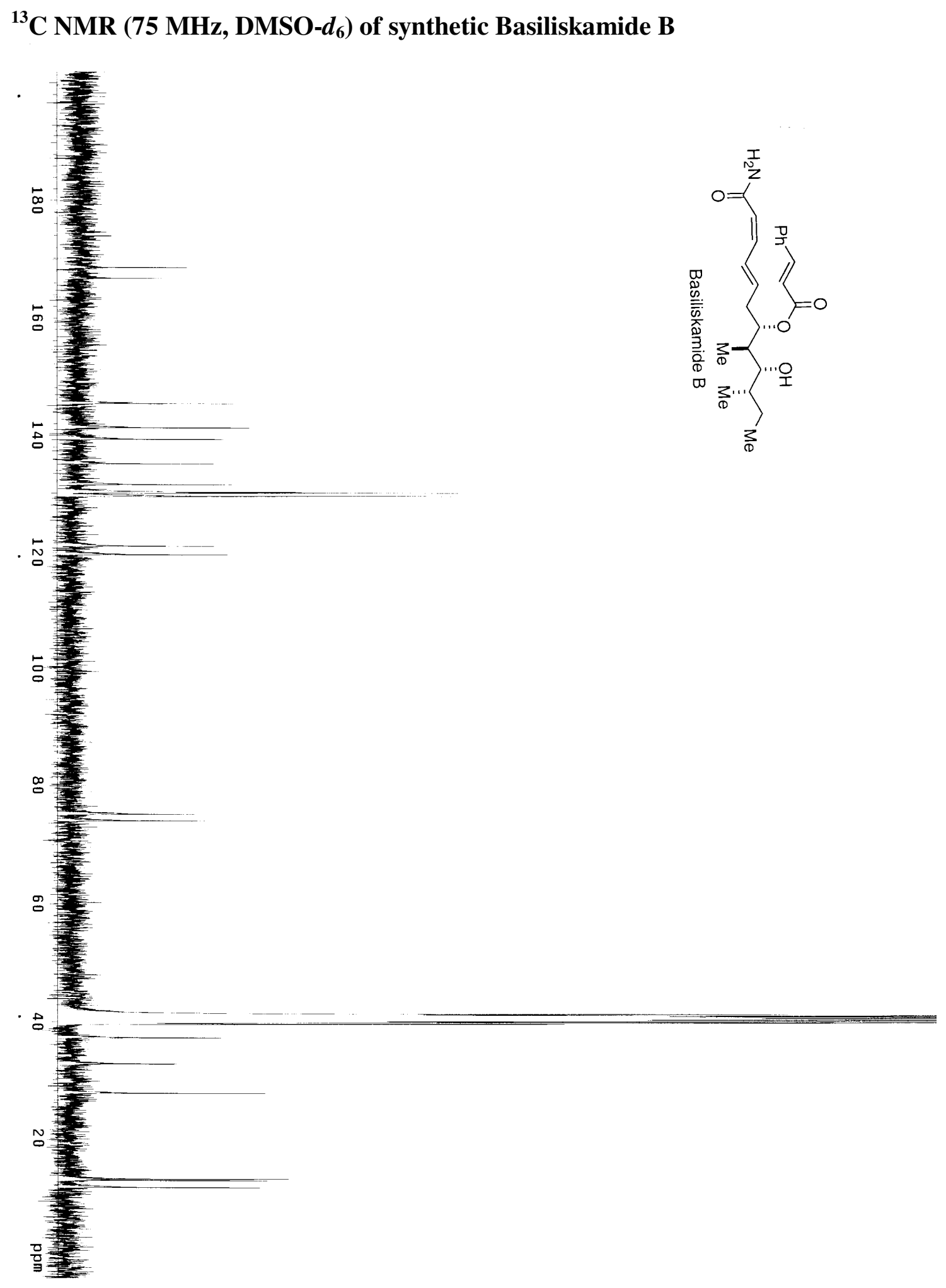




\section{${ }^{13}$ C NMR (100 MHz, DMSO- $\left.d_{6}\right)$ of natural Basiliskamide B}

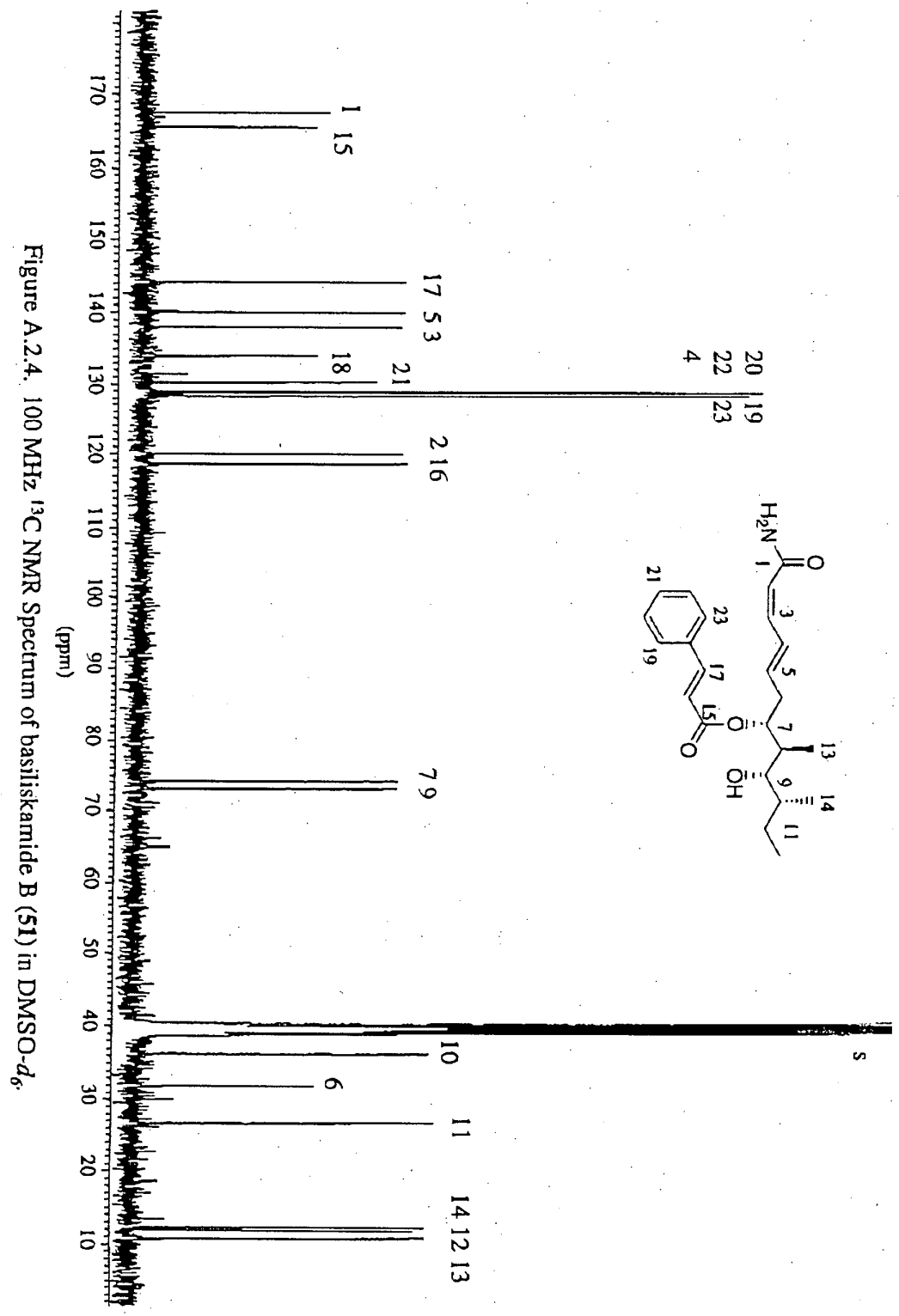

